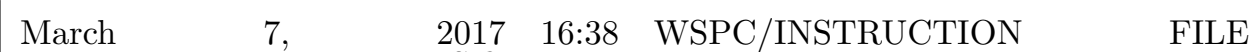
PressureWaves-ijam revised ${ }^{\circ}{ }^{2}{ }^{\circ}$ final

International Journal of Applied Mechanics

(C) Imperial College Press

\title{
PRESSURE WAVE IN LIQUID GENERATED BY PNEUMATIC PISTONS AND ITS INTERACTION WITH A FREE SURFACE
}

\author{
VICTORIA SUPONITSKY, DAVID PLANT \\ General Fusion Inc., 108-3680 Bonneville Place,Burnaby, BC V3N 4T5, Canada \\ ELDAD J AVITAL, ANTE MUNJIZA \\ School of Engineering and Materials Science, Queen Mary University of London, Mile End \\ Road E14NS, London, UK \\ Received date \\ Accepted date
}

\begin{abstract}
Numerical analysis of a pressure wave generated in a liquid $\mathrm{Pb}$ upon impact of the pneumatic pistons and its interaction with a free-surface has been performed for the geometry and parameters of the plasma compression system prototype constructed by General Fusion Inc. Stress wave developing in the hammer-anvil piston assembly is first simulated using high fidelity structural mechanics research code, then propagated through the liquid $\mathrm{Pb}$ with several solvers within OpenFOAM ${ }^{\circledR}$ software and also with non-linear acoustics in-house code based on the Westervelt equation. In the current system, a pressure wave transmitted into the liquid $\mathrm{Pb}$ is characterized by a complex temporal double peak structure and strong spatial amplitude variation. An imprint of discrete pulses remains detectable during the entire propagation of the combined wave. An excellent agreement between the results produced with different numerical codes is obtained. Non-linear effects associated with equation of state are found to be significant at impact velocities of $50 \mathrm{~m} / \mathrm{s}$, while at lower velocities of $10 \mathrm{~m} / \mathrm{s}$ the difference between the results obtained with linear and non-linear equations of state is negligible. Liquid-gas interface dynamics during the compression process of a spherical gas cavity is captured very well by the "compressibleInterFoam" within OpenFOAM.
\end{abstract}

Keywords: Pressure waves; non-linear acoustics; impact dynamics; multiphase flow.

\section{Introduction}

Current work aims to develop a numerical framework to study pressure waves propagation in liquids, their interaction with a free surface, and a method to generate those waves by a longitudinal impact of a pneumatic piston (or a piston assembly). Using a pneumatic piston to generate a pressure wave in a liquid is relatively simple and straightforward. However, spatial and temporal structure of the generated pulse, details of its transmission across solid-liquid interface and overall energy transfer efficiency are far from simple and strongly influenced by different parameters such as materials and geometry of the assembly. This particular work has been driven by the necessity to develop a reliable set of numerical tools to study hydrodynamics of compression of magnetized plasma by a liquid metal liner for configuration and pa- 
March 7, $2017 \quad 16: 38$ WSPC/INSTRUCTION FILE PressureWaves-ijam revised ${ }^{\circ} \mathrm{VS}{ }^{\circ}$ final

rameters relevant to the recently proposed Magnetized Target Fusion (MTF) scheme [Laberge, 2009] that is currently pursued by General Fusion Inc. and is discussed in more details later on. Although majority of the obtained results are related to the above-mentioned specific application, physical phenomena studied and numerical tools developed are relevant and can be further applied to study many different fundamental and applied problems, some of which are discussed below.

In the context of the application in question, a pneumatic piston assembly consists of a thick solid cylinder impacting upon another thick cylinder which is placed in contact with a liquid metal. The problem of stress propagation in cylindrical bars upon the longitudinal impact has been extensively studied in relation to Hopkinson bar material tests experiments to examine material properties under dynamic load, see for example [Nemat-Nasser et al., 1991] for a broad review. Over the years a number of modifications to the basic idealized analytical one-dimensional model have been proposed in order to achieve better agreement with experimental data [e.g. Meyers, 1994, Vales et al., 1996]. The majority of the work, however, concentrated on high aspect ratio, long, cylindrical bars. Pressure waves in liquids are encountered in number of practical applications such as hydraulic bars and dampers, drilling hammers, free piston compression shock tubes, pipelines (waterhammer phenomena) etc. They are also relevant to biological flows applications, e.g. shock waves focusing in lithotripsy [Takayama, 1993]. There are some fundamental differences in the behavior of compressible gases and liquids, e.g. ability of liquid to undergo a phase change (cavitate) when subjected to tension. From a computational point of view, depending on the application and importance of detailed understanding of cavitation, computational models of various complexity can be used, see for example [Charriere et al., 2015 ] for a recent comparative study. Equation of state should be also tuned for each liquid considered.

Numerical tools capable of simulating interaction of a pressure wave propagating in a liquid with a free surface and subsequent dynamics of a liquid-gas interface can be applied to study many fundamental and applied problems such as: (i) liquid-gas interface instabilities (Richtmyer-Meshkov, Bell-Plesset and Rayleigh-Taylor), (ii) shock induced bubble collapse in liquid [Johnsen and Colonius, 2008], (iii) cavitation zones originated from underwater explosion near the free surface [Ichihara et al., 2009, Kedrinskii, 2005], (iv) air bubble curtains to attenuate pressure waves propagating through the liquid [Tsouvalas and Metrikine, 2016].

In this work the focus is on hydrodynamics of plasma compression by a liquid metal liner driven by a converging pressure wave initiated by pneumatic pistons [Laberge, 2009]. Compression system in question consists of a steel vessel filled with a rotating liquid metal (lead-lithium eutectic) such that the evacuated cavity is formed inside the vessel. Deuterium-tritium magnetized plasma is delivered into this cavity. Pneumatic steel pistons mounted on the outer surface of the vessel generate an acoustic pulse that is transmitted into the liquid metal upon the impact. The transmitted pulse then propagates inwards and is amplified by a geometric con- 
March 7, $2017 \quad 16: 38$ WSPC/INSTRUCTION FILE

PressureWaves-ijam revised ${ }^{\circ}{ }^{2}{ }^{\circ}$ final

vergence factor determined by the specific shape of the vessel, pistons' arrangement and timing. When the pressure wave reaches the liquid-plasma interface and interacts with it, the interface undergoes rapid inward acceleration and travels towards the center compressing the plasma [Richardson et al., 2013]. It should be noted, that the pressure wave is almost entirely reflected from the liquid-plasma interface as a rarefaction wave, which then subjects liquid to tension at some distance from the interface. In the ideal case, the energy stored in the pressure pulse is converted into kinetic energy of the detached liquid metal shell that is separated from the rest of the fluid by the cavitation/foam region. This detached shell is the one that ultimately compresses the plasma inside the evacuated cavity. Geometry of the detached shell depends strongly on the shape of the pressure wave at the interface, which in turn depends on geometry of the piston assembly and arrangement of the pistons. An experimental work primarily dedicated to study controlled cavitation and formation of detached shell in converging geometry, is an additional ongoing work in progress [Huneault and Higgins, 2015].

An ultimate goal of this ongoing study is to design an energy efficient plasma compression system, therefore energy transfer between different components of the system is of great importance. As such, details of the stress wave propagation inside the piston assembly and transmission of the stress wave across solid-liquid interface are important parts of the overall problem. It should be also noted that the converging wave in liquid is combined from a discrete pressure waves generated by each of the pneumatic pistons. The structure of this combined wave and, in particular, the degree of uniformity of the wave front as it approaches the liquid-plasma interface is also of interest as non-uniformities might seed hydrodynamic instabilities, such as, Richtmyer-Meshkov [Suponitsky et al., 2014] and eventually compromise compression efficiency.

Simulations have been carried out for the geometry and parameters of the existing prototype of compression system which is described in detail in Section 2. Interaction of the plasma and the liquid metal and magnetic effects are omitted at this stage. Collapse dynamics of a spherical cavity, that could be expected in the current prototype under "perfect" conditions of simultaneous pistons impact and initially smooth and symmetric liquid-gas interface, is studied and discussed. Some preliminary results and validation tests can be found in our earlier work [Suponitsky et al., 2014, Suponitsky et al., 2015]. The rest of the article is organized as follows. The methodology and problem statement are described in Section 2, in which numerical details of structural, acoustic and fluid dynamics simulations are described, respectively, in subsections 2.1, 2.2 and 2.3. The results are presented in Section 3, where stresses developing in the piston assembly upon the impact are studied in subsection 3.1, propagation of the pressure pulse in the liquid metal is discussed in subsection 3.2 and dynamics of a spherical cavity collapse is presented in subsection 3.3. Finally, summary and main conclusions are given in Section 4. 
March 7, $2017 \quad 16: 38$ WSPC/INSTRUCTION FILE PressureWaves-ijam revised ${ }^{\circ} \mathrm{VS}{ }^{\circ}$ final

Suponitsky, Plant, Avital, Munjiza

\section{Methodology and Problem Statement}

The first experimental compression system prototype consists of fourteen pneumatic steel pistons mounted on $20 \mathrm{~cm}$ thick steel sphere with inner radius of $0.5 \mathrm{~m}$ (Fig. 1). Pistons with an operating velocity range of between 10 and $50 \mathrm{~m} / \mathrm{s}$, are arranged in two staggered rings of seven pistons below and above the equator (at the angle of $22.5^{\circ}$ ). Interior of the sphere is filled with tangentially pumped molten $\mathrm{Pb}$ such that evacuated cavity is formed inside the sphere. Rotational velocity of the liquid inside the sphere is relatively small compared to that of wave propagation $(10 \mathrm{~m} / \mathrm{s}$ compared to $1750 \mathrm{~m} / \mathrm{s}$ ) and is omitted in this study for the sake of simplicity. To evaluate capability of the current numerical setup to capture interface dynamics, collapse of an unperturbed spherical gas cavity is studied here for simplicity.

The following aspects of pressure wave formation and subsequent evolution are studied: structure of the wave inside the piston assembly, transmission of the wave between steel and liquid $\mathrm{Pb}$, structure of the combined wave as it propagates through the liquid $\mathrm{Pb}$ towards the cavity and, finally, a prediction of cavity collapse characteristics resulting from interaction between the pressure wave and the gas cavity. The entire problem is sub-divided into several computational stages and the output of one stage is used as an input to the next stage. Formation and structure of the pressure wave developing inside the individual steel piston is studied using the high fidelity research structural code "Y"-code which is based on a novel finite-discrete element approach [Munjiza, 2005]. The obtained pressure wave is then used as a boundary condition into fluid and acoustic solvers to study its propagation in liquid $\mathrm{Pb}$. Fluid simulations are performed using "sonicLiquidFoam", "cavitatingFoam" and "compressibleInterFoam" solvers within open source $\mathrm{C}++$ libraries of OpenFOAM [CFD Direct, http://cfd.direct]. The same pressure wave is also propagated using the in-house non-linear acoustic solver based on Westervelt equation [Avital et. al., 2010, Avital et al., 2013] and the results are compared to each other. To follow the process of gas cavity collapse "compressibleInterFoam" solver of OpenFOAM is used. It employs the Volume of Fluid (VoF) phase-fraction-based interface-capturing approach that is suitable for modeling two compressible and immiscible fluids.

\subsection{Problem statement for the structural simulations with "Y"-code}

Structural simulations have been carried out using "Y"-code [Munjiza, 2005]. Numerical implementation of the code can be found in [Munjiza and Andrews, 2000, Munjiza and Latham, 2004], while validation tests and applications in [Latham et al., 2008, Rougier et al., 2014]. In current assembly, each of the pneumatic steel pistons consists of a "hammer" piston and floating "anvil" piston. A hammer piston accelerated by compressed air to velocities of up-to $50 \mathrm{~m} / \mathrm{s}$, impacts an anvil piston which is placed in contact with liquid $\mathrm{Pb}$. Upon the impact a stress wave starts to propagate through the anvil and hammer pistons. Once the wave in the anvil reaches the steel-liquid $\mathrm{Pb}$ interface, part of it is transmitted into the liquid 
$\begin{array}{lllll}\text { March } & 7, & 2017 & 16: 38 & \text { WSPC/INSTRUCTION }\end{array}$

PressureWaves-ijam revised ${ }^{\circ} \mathrm{VS}{ }^{\circ}$ final

$\mathrm{Pb}$ and part is reflected back depending on the mismatch of acoustic and geometric impedances (material properties used in current simulations are listed in Table 1).

Nominal numerical setup consists of three bodies as shown in Fig. 2a, where a hammer piston with an assigned initial velocity, impacts an anvil that is initially at rest and placed in contact with large volume of liquid $\mathrm{Pb}$. This setup is aimed to obtain the structure of the pressure wave exerted upon the liquid $\mathrm{Pb}$ as a result of the piston impact in the current assembly. Elastic solid material model has been used for the hammer and anvil steel pistons. Liquid $\mathrm{Pb}$, on the other hand, is modeled using the so called Lagrangian approach. This is achieved by simply setting material properties of liquid $\mathrm{Pb}$ into the non-linear elastic equation of state which connects logarithmic strains to Cauchy stress through elastic constants. By setting deviatoric part to zero, only spherical, i.e. pressure part remains. To this is added a linear relationship between strain rate and corresponding Cauchy stress, i.e. viscosity. This approach is valid as long as the particle displacements are small enough not to distort the finite element mesh, otherwise one would need remeshing, which is not done. "Y"-code has constitutive law in terms of Lamé constants $\lambda$ and $\mu$ as:

$$
\sigma_{i j}=2 \mu \varepsilon_{i j}+\lambda \varepsilon_{i i}
$$

By setting $\mu=0$ and $\lambda=\kappa$ (where $\kappa$ is a bulk modulus) one obtains zero shear. Extensive set of test cases has been carried out and the results obtained with "Y"code have been validated against those obtained with commercial structural codes such as ANSYS and LS-Dyna.

In the current assembly both hammer and anvil pistons have a cylindrical shape with radius $0.15 \mathrm{~m}$. A small flair is added to the anvil near the surface contacting liquid $\mathrm{Pb}$ that increases its radius to $0.17 \mathrm{~m}$ (Fig. 2b). Lengths of hammer and anvil pistons are $0.182 \mathrm{~m}$ and $0.185 \mathrm{~m}$, respectively. Liquid $\mathrm{Pb}$ tank is also a cylinder with length and radius equal to $0.6 \mathrm{~m}$. Isotropic tetrahedral nearly uniform mesh has been used in majority of simulations and a typical mesh is shown in Fig. 2b. For a nominal case tetrahedrons with edge length of $5 \mathrm{~mm}$ have been used for hammer and anvil parts, while for a lead tank the edge length has been increased to $6 \mathrm{~mm}$. This resulted in a computational mesh with about 17.2 million elements where the majority of the cells are placed in the $\mathrm{Pb}$ tank part.

Table 1: Material Properties ( $\lambda$ and $\mu$ are Lamé constants)

\begin{tabular}{lllll}
\hline Material & $\rho\left[\mathrm{kg} / \mathrm{m}^{3}\right]$ & $c_{\mathrm{S}}[\mathrm{m} / \mathrm{s}]$ & $\lambda[\mathrm{Pa}]$ & $\mu[\mathrm{Pa}]$ \\
Steel & 7750 & 4684 & $9.8 \times 10^{10}$ & $6.54 \times 10^{10}$ \\
Lead ("Y"-code) & 10530 & 1750 & $4.6 \times 10^{10}$ & 0 \\
Lead (OF) & 10650 & 1770 & - & - \\
\hline
\end{tabular}


March 7, $2017 \quad 16: 38$ WSPC/INSTRUCTION $\quad$ FILE

PressureWaves-ijam revised ${ }^{\circ} \mathrm{VS}{ }^{\circ}$ final

Suponitsky, Plant, Avital, Munjiza

\subsection{Problem statement for the fluids non-linear acoustics simulations}

The Westervelt equation which is of second order non-linearity has been simulated for the propagation of the pressure pulse through the liquid $\mathrm{Pb}$. The equation is [Hamilton and Blackstock, 1998, Avital et al., 2013]:

$$
\nabla^{2} p-\frac{1}{c_{\mathrm{Pb}}^{2}} \frac{\partial^{2} p}{\partial t^{2}}+\frac{\beta_{\mathrm{Pb}}}{\rho_{\mathrm{Pb}} c_{\mathrm{Pb}}^{4}} \frac{\partial^{2} p^{2}}{\partial t^{2}}+\frac{\delta_{\mathrm{Pb}}}{c_{\mathrm{Pb}}^{4}} \frac{\partial^{3} p}{\partial t^{3}}=0 .
$$

In Eq. $2 p$ is the pressure fluctuation and $\beta_{\mathrm{Pb}}$ is the non-linear coefficient taken as $1+B /(2 A)$, where $B / A$ is a measure of the deviation of the local speed of sound from its ambient value. Taking $\beta_{\mathrm{Pb}}=1$ yields the linear equation of state (Eq. 4) which is used in OpenFOAM simulations and discussed in greater details in Section 2.3. Commonly the Ballou's statistical rule of $B / A=1.2 \times 10^{4} / c_{\mathrm{Pb}}-0.5$ can be used for liquid metals, where $c_{\mathrm{Pb}}$ is measured in $\mathrm{m} / \mathrm{s}$ [Avital et al., 2013]. $\delta_{\mathrm{Pb}}$ is the diffusivity coefficient that is taken as $\nu_{\mathrm{Pb}}\left[4 / 3+\mu_{\mathrm{B}, \mathrm{Pb}} / \mu_{\mathrm{Pb}}+\left(\gamma_{\mathrm{Pb}}-1\right) / P r_{\mathrm{Pb}}\right]$, where $\mu_{\mathrm{B}, \mathrm{Pb}} / \mu_{\mathrm{Pb}}$ is the ratio between the second to first viscosity coefficients and $\left(\gamma_{\mathrm{Pb}}-1\right) / P r_{\mathrm{Pb}}$ is the heat conduction effect that is of less importance for liquids [Avital et al., 2013]. Numerical experimentation showed that the diffusion term had little effect on the pulse propagation due to its short time scale.

The Westervelt equation is of second order in its non-linearity, thus neglecting third order terms density fluctuations of $\mathcal{O}\left(\left(\rho^{\prime} / \rho_{\mathrm{Pb}}\right)^{3}\right)$. The calculations in Section 3 confirm the validity of this assumption. The second-order Lagrangian density term is also neglected, which means that the accumulative non-linear propagation effects are assumed to be of higher importance than the local non-linear effects. To check this assumption, a correction was added to the pressure $p$ as follows [Avital et al., 2013].

$$
p_{\mathrm{r}}=p+\frac{\rho_{\mathrm{Pb}}}{4}\left(\nabla^{2}+\frac{1}{c_{\mathrm{Pb}}^{2}} \frac{\partial^{2}}{\partial t^{2}}\right) \varphi^{2}, p=-\rho_{\mathrm{Pb}} \frac{\partial \varphi}{\partial t} .
$$

Using scaling analysis, one can see that $p_{\mathrm{r}}=p\left[1+\mathcal{O}\left(p /\left(\rho_{\mathrm{Pb}} c_{\mathrm{Pb}}^{2}\right)\right)\right]$. Taking $p=1.5 \mathrm{GPa}$ which is the highest pressure considered at the anvil, yields $\mathcal{O}\left(p /\left(\rho_{\mathrm{Pb}} c_{\mathrm{Pb}}^{2}\right)\right)=0.045$ thus justifying the assumption of dominance of accumulative non-linear effects over local ones at least near the anvil.

Equation 2 was discretized using second order finite difference schemes on spherical co-ordinates. A Crank-Nicolson or full implicit discretization was used in the radial direction to better deal with the geometrical amplification caused by the converging wave. Both methods showed similar results. Explicit discretization was used in the two other spatial directions. Grid stretching was used to cluster points near the equator to increase resolution near the anvils and reduce the CFL limit caused by the fine spherical grid near the poles. Zero pressure gradient was taken 
March 7, $2017 \quad 16: 38$ WSPC/INSTRUCTION FILE

PressureWaves-ijam revised ${ }^{\circ} \mathrm{VS}{ }^{\circ}$ final

on the steel wall and pressure values on the anvil were extrapolated from the structural simulations. The interface of the liquid lead with the inner cavity was modeled as a free surface. A grid size of $(501,385,129)$ points in the radial, azimuthal and zenithal directions was used respectively. Increasing the grid resolution to $(501,581$, 193) points had a small effect on the solution. The numerical schemes were verified against linear and non-linear propagation test cases.

\subsection{Problem statement for the fluid simulations with OpenFOAM}

Three different OpenFOAM solvers ( "sonicLiquidFoam", "compressibleInterFoam" and "cavitatingFoam") suitable for computation of a compressible liquid are used in this study. "sonicLiquidFoam" is a single phase solver used to propagate pressure waves in the liquid $\mathrm{Pb}$. It is a 2nd order of accuracy transient solver that uses PISO (Pressure Implicit with Splitting of Operators) to march forward in time. The "compressibleInterFoam" is a two-phase compressible solver that is used to study wave propagation in the liquid $\mathrm{Pb}$ and to follow dynamics of a gas cavity collapse resulting from interaction between pressure wave and a liquid-gas interface. Handling of a free-surface evolution in "compressibleInterFoam" is similar to that of "interFoam" solver which has been extensively applied and validated [Rusche, 2002, Deshpande et al., 2012, Suponitsky et al., 2014]. "cavitatingFoam" solver is mainly used for the sake of completeness as it employs similar numerics for the liquid phase as in both "compressibleInterFoam" and "sonicLiquidFoam" solvers. It is used for validation purposes and to explore potential development of cavitated $\mathrm{Pb}$ regions during the pulse propagation through the liquid $\mathrm{Pb}$.

It is important to discuss equation of state available for the liquid phase in OpenFOAM solvers as it is studied considerably less compared to that in gases. In all up-to-date versions of OpenFOAM the only equation of state available in "sonicLiquidFoam" and "cavitatingFoam" solvers is a linear barotropic equation of state, which is given by the following formula:

$$
\rho=\rho_{0}+\psi\left(P-P_{0}\right)
$$

The variable $\psi$ represents the derivative of density with respect to pressure and assumed constant. $\left(\rho_{0}, P_{0}\right)$ is a reference state and both reference density and pressure are constants. In extensively tested and validated OpenFOAM version v1.7 which is mainly used in this work, "compressibleInterFoam" solver also uses linear equation of state (Eq. 4) and assumes constant fluid temperature, i.e. no energy equation is solved. In more recent versions an energy equation has been added and more choices for equation of state have become available. To investigate non-linear effects, some of the simulations have been re-run using version v4 with adiabatic stiffened gas equation of state in the form of:

$$
\rho=\rho_{0}\left(\frac{P+B}{P_{0}+B}\right)^{1 / \gamma}
$$




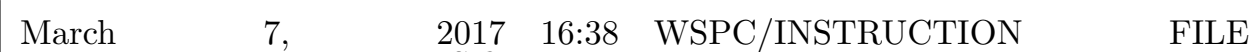
PressureWaves-ijam revised ${ }^{\circ} \mathrm{VS}{ }^{\circ}$ final

where $B=5.055 \times 10^{9} \mathrm{~Pa}$ and $\gamma=6.6$ for the liquid $\mathrm{Pb}$. These values yield the same speed of sound $c_{\mathrm{Pb}}$ and non-linear coefficient $\beta_{\mathrm{Pb}}$ as used for the Westervelt equation (Eq. 2). It is worth mentioning that Eq. 4 implies linear relation between density and pressure and, therefore, it is accurate when density perturbations associated with pressure wave propagation through the liquid, remain small. To estimate amplitude of density fluctuations at which non-linear effects become important, results are compared with those obtained with non-linear acoustic solver based on Westervelt equation [Punekar et al., 2010] and also with those obtained with v4 version of "compressibleInterFoam" and adiabatic stiffened gas equation state (Eq. 5).

The computational model consists of a $1 \mathrm{~m}$ diameter sphere with fourteen round patches (curved and aligned with surface of the sphere or flat and slightly extruded), which represent pistons with radius $17 \mathrm{~cm}$, each. "Pistons" are arranged in two staggered rings of seven as in experimental setup in Fig. 1a. A pressure pulse obtained in the structural simulations is imposed as a time-dependent boundary condition at piston patches. Each "piston" patch is divided into ten segments in the radial direction and averaged pulse is then applied to each segment. A zero-gradient boundary condition is set for the velocity at the "piston" patch allowing some mass influx into the domain. Once the whole pressure pulse enters the domain, boundary conditions are switched to zero velocity and zero pressure gradient as for all other boundaries.

A computational mesh used to study wave propagation is shown in Fig. 3(a). In those simulations only $\frac{1}{7}$ part of a sphere has been simulated. Periodic boundary condition has been applied to ensure repeatability of the pattern. A hybrid mesh has been used in the current setup. An unstructured block is used near the outer surface of the sphere to incorporate circular piston patches on an otherwise structured mesh. A central part of the sphere has been excluded from simulations to avoid singularity and very small grid cells near the center. A structured spherical block has uniform grid points distribution with number of points equal to $n_{\mathrm{r}}=400, n_{\theta}=400$ and $n_{\phi}=80$ in the radial, azimuthal and zenithal directions, respectively. To follow process of cavity collapse, the entire sphere with all fourteen pistons is simulated and a circular flat piston patches are extruded from the surface of the sphere as shown in Fig. 3(b). A hybrid mesh is used also in those simulations. Unstructured mesh is used for extruded pistons, outer portion of the sphere and a central part of the sphere. The rest of the sphere is filled with structured orthogonal mesh. A structured block has uniformly spaced grid points with corresponding number of grid points in the radial, azimuthal and zenithal directions equal to $n_{\mathrm{r}}=200, n_{\theta}=300$ and $n_{\phi}=200$. Entire computational mesh consists of about 18 million cells. A gas cavity of a spherical shape with a diameter of $10 \mathrm{~cm}$ has been prescribed as initial condition to mimic a plasma target in the compression system. Also shown in Fig. 3(b) are horizontal (red color) and vertical (blue color) planes, which we are going to refer to when presenting and analyzing results in Section 3. Paths along "Profile Lines", marked by the color green in Fig. 3(b), are used for plotting pressure profiles during the wave propagation. Numerical schemes with second order 
March 7, $2017 \quad 16: 38$ WSPC/INSTRUCTION FILE

PressureWaves-ijam revised ${ }^{\circ} \mathrm{VS}{ }^{\circ}$ final

of accuracy in space and time have been employed in all OpenFOAM simulations. Calculations have been run on a local cluster using 12 to 24 cpus (Intel Xeon E52640v2 2.0GHz CPUs). Simulations have been performed for liquid $\mathrm{Pb}$ and gas with following properties $\rho_{\mathrm{Pb}}=10650 \mathrm{~kg} / \mathrm{m}^{3}, c_{\mathrm{Pb}}=1770 \mathrm{~m} / \mathrm{s}, \nu_{\mathrm{Pb}}=1.38 \times 10^{-7} \mathrm{~m}^{2} / \mathrm{s}$ and $\rho_{\text {gas }}=1 \mathrm{~kg} / \mathrm{m}^{3}, c_{\text {gas }}=316 \mathrm{~m} / \mathrm{s}$ and $\nu_{\text {gas }}=1.48 \times 10^{-5} \mathrm{~m}^{2} / \mathrm{s}$.

\section{Results}

\subsection{Structural "Y"-code Simulations}

In this section we show the results of the stress wave developing in the piston assembly upon the impact, with the main objective to extract temporal and spatial structure of a pressure pulse transmitted into liquid $\mathrm{Pb}$. A cross-section of a stress wave propagating in the hammer-anvil system (before the wave begins its transmission into a liquid $\mathrm{Pb}$ ) is shown by the contours of the axial stress $\sigma_{x x}$ in Fig. 4 for a $50 \mathrm{~m} / \mathrm{s}$ impact, where time $\mathrm{t}=0$ corresponds to the moment the hammer gets in contact with the anvil. One can see the development of symmetric compressive wave (before wave reaches the flair) in both the hammer and anvil pistons. It can be also seen that the stress wave develops a complex temporal and spatial structure which differs significantly from the one expected from a simplified model based on uniaxial stress assumption. Based on 1D analysis of a thin cylindrical bar being impacted with a cylindrical projectile of length $L$, one would expect a rectangular pulse of length $2 L$ propagating without distortion through the bar, if the bar and projectile are of the same material. Stress $\sigma$ generated by the impact at velocity $V$, a particle velocity $U_{\mathrm{p}}$ and a velocity of a longitudinal wave in a thin bar $C_{0}$ are then correspondingly given by Eq. 6 [e.g. Meyers, 1994]:

$$
\sigma=\frac{1}{2} \rho C_{0} V, \quad U_{p}=\frac{V}{2}, C_{0}=\sqrt{\frac{E}{\rho}} .
$$

For our set of parameters the values expected from uniaxial model (Eq. 6) are:

$$
\sigma=\sigma_{x x} \approx 0.9 \mathrm{GPa} ; U_{\mathrm{p}}=25 \mathrm{~m} / \mathrm{s}, C_{0}=4684 \frac{\mathrm{m}}{\mathrm{s}} .
$$

Deviations from uniaxial model happen because in the real case there is a radial inertia and a wave interaction with the external surfaces of the cylindrical piston. Radial inertia is caused by the kinetic energy of the material flowing radially outward as the bar is compressed because of a non-zero Poisson ratio of the material. By

reducing Poisson ratio $\nu$ from 0.3 to 0 (uniaxial case) results of $1 \mathrm{D}$ model given by Eq. 6 have been fully recovered in our simulations.

At early stages of a wave propagation (before wave reaches steel-liquid $\mathrm{Pb}$ interface), the problem can be reduced to that of wave propagation in a thick cylindrical bar (we ignore effect of the flair for the sake of simplicity). Immediately after the impact, a plane pressure dilatation wave starts propagating through the pistons 
March 7, $2017 \quad 16: 38$ WSPC/INSTRUCTION FILE PressureWaves-ijam revised ${ }^{\circ} \mathrm{VS}{ }^{\circ}$ final

(hammer and anvil) with velocity of the dilatational wave in unbounded medium $C_{1}$ given by:

$$
C_{1}=\sqrt{(\lambda+2 \mu) / \rho} \approx 5400 \mathrm{~m} / \mathrm{s},
$$

where $\lambda$ and $\mu$ are Lamé constants. Behind this wave front, the same uniform state of stress, as resulted from the impact, occurs (Fig. 4a). The size of this region, however, is getting smaller because of unloading dilatational waves originated from the points lying on circular edges of colliding faces of the cylinders and also traveling with velocity $C_{1}$ as can be seen in Figs. 4(b) and (c). The unloading dilatation waves are in turn followed by shear waves propagating from the same points at velocity $C_{2}$ given by:

$$
C_{2}=\sqrt{\mu / \rho} \approx 2900 \mathrm{~m} / \mathrm{s} .
$$

Those waves are also seen in Figs. 4(b) and (c).

It is worth noting that for the current geometry of the piston assembly, the entire stress propagation inside the anvil belongs to the near-field and short-time response, therefore strong deviation from the 1D model is not surprising, as it is mostly suitable for the far-field and long-time response. If the dimensional time for the snapshots presented in Fig. 4 is normalized by the characteristic time $\mathrm{t}_{0}=\mathrm{R} / C_{1}$ (time it takes for the wave with velocity $C_{1}$ to travel distance equal to the radius of the bar) we get $\mathrm{t} / \mathrm{t}_{0}=0.2,0.72$ and 1.3 , respectively, for parts (a), (b) and (c) of the figure, clearly indicating near-field stress distribution. More details about near-field and short-time response of a wave propagation upon longitudinal impact of two thick cylindrical bars can be found in the theoretical paper of [Vales et al., 1996].

Propagation of the stress pulse across steel-liquid $\mathrm{Pb}$ interface is shown in Fig. 5 by the contours of axial stress at four different time instances $(t=76,96,136$ and $170 \mu \mathrm{s})$ for $50 \mathrm{~m} / \mathrm{s}$ impact. Only the compressive part of the stress wave is shown in Fig. 5, i.e. only contours with negative axial stresses are plotted. It takes the pulse about $35 \mu$ s to travel through the anvil and reach the interface with liquid $\mathrm{Pb}$, while the duration of the entire pulse is approximately $70 \mu \mathrm{s}$. As such, instances shown in Fig. 5 correspond approximately to: half of the pulse being transmitted into the $\mathrm{Pb}$ (part a), three-quarters of the pulse is in the $\mathrm{Pb}$ (part b), entire pulse is in the $\mathrm{Pb}$ (part $\mathrm{c}$ ) and the transmitted compressive pulse propagates through the volume of liquid $\mathrm{Pb}$ (part d). For the piston geometry in question, the transmitted wave is strongly non-uniform across the anvil face, with some stress concentration clearly visible in the flairing section. Stress waves of considerable amplitude continue to bounce inside both anvil and hammer pistons long after the entire pulse has been transmitted into the liquid $\mathrm{Pb}$.

It is worth noting that in the computational model the hammer, anvil and the liquid $\mathrm{Pb}$ are specified as three separate parts. Therefore, if the interface between anvil and liquid $\mathrm{Pb}$ is subjected to tension at some point during the simulation (for example, due to the reflections back into the anvil), the liquid $\mathrm{Pb}$ may separate from the anvil. In the real case this might indicate development of the cavitating 


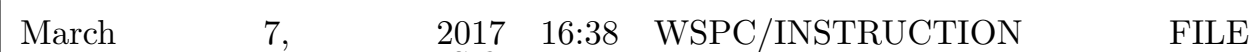

PressureWaves-ijam revised ${ }^{\circ} \mathrm{VS}{ }^{\circ}$ final

$\mathrm{Pb}$ region in the vicinity of anvil face. With current numerical setup, continuity of the axial stress and velocity across the interface is expected as long as parts remain in contact (axial direction corresponds to the direction normal to the interface unless anvil face is getting deformed as it penetrates into liquid $\mathrm{Pb}$ ). Therefore, discontinuity in the axial stress component observed at later times (Fig. 5c, d) indicates separation between the parts. Separation mainly occurs at the center of the anvil face, while away from the center, in the area of the flair, anvil and liquid $\mathrm{Pb}$ remain in contact. Separation between steel and lead may have long lasting consequences. In the system in question, it occurs after the entire pulse has been transmitted into the liquid $\mathrm{Pb}$ and, therefore, the damage is likely to be limited to the cavitation damage at the anvil face.

Results obtained with different piston geometries indicate that for certain geometries separation may also occur during the pulse transmission. In this case, energy transmission into the liquid $\mathrm{Pb}$ is interrupted once the parts separate and the remaining portion of the pulse is reflected back into the anvil. This situation is highly undesirable as it reduces overall energy efficiency of the system. It is convenient to examine possible separation between the parts by following the axial displacement and axial velocity at the mesh nodes on both sides of the interface, as it is shown in Figs. 6a \& b for the nodes positioned at the center of the anvil face. Solid and broken lines correspond to the nodes in the steel and liquid $\mathrm{Pb}$, respectively. One can see that displacement and velocity on the both sides of the interface follow each other up to about $130 \mu$ s (entire pulse has been transmitted into Pb by then) and depart afterwards (compare solid and broken lines).

Time history of the stress components at several axial positions along the anvil are plotted in Fig. 7. Parts (a) and (b) of the figure show axial $\sigma_{x x}$ and radial $\sigma_{r r}$ stress components, respectively. Axial positions are normalized by the length of the anvil, where $\mathrm{x}=0$ and $\mathrm{x} / L_{\text {anvil }}=1$ correspond to the ends facing hammer and liquid $\mathrm{Pb}$, respectively. Solid lines show results in the center of the anvil $\mathrm{r} / \mathrm{R}=0$, while broken lines added in part (a) correspond to the points at the edge $\mathrm{r} / \mathrm{R}=1$. Time history of the radial displacement at the anvil surface $(r / R=1)$, for the same axial positions along the anvil is plotted Fig. 7(c). It can be seen that compressive stress state resulting directly from the impact holds for a certain period of time (about $25 \mu \mathrm{s}$ ) at the axial positions close the hammer-anvil interface. During this time both axial and radial stresses are negative and nearly constant (solid red lines in Figs. 7a and b). At the positions further along the anvil, time duration of nearly constant stresses decreases and disappears by $\mathrm{x} / L_{\text {anvil }}=0.75$ (blue, green and black lines up-to about $35 \mu \mathrm{s}$ ). One can also see that initially (up to about $50 \mu \mathrm{s}$ for the position closest to the hammer) the anvil undergoes radial expansion due to the radial inertia caused by the kinetic energy of the material flowing radially outward as the bar is compressed (Fig. 7c). As a result, the amplitude of the compressive radial stresses starts to decrease causing a temporary decrease in the amplitude of the axial compressive stresses as well. At some point radial stresses reverse from compressive 
March 7, $2017 \quad 16: 38$ WSPC/INSTRUCTION FILE PressureWaves-ijam revised ${ }^{\circ} \mathrm{VS}{ }^{\circ}$ final

to tensile as the anvil continues to expand. Once radial stresses become tensile, amplitude of the axial compressive stresses starts to increase again, and reaches its local maximum at the time of the maximum radial expansion. For example, at $\mathrm{x} / L_{\text {anvil }}=0.75$ maximum radial expansion occurs at about $70 \mu \mathrm{s}$ (black line at Fig. 7c) which roughly corresponds to the maximum tensile radial stress (black line at Fig. 7b) and also to the time of the second peak in the axial compressive stress (black line at Fig. 7a). One can also note that the maximum radial compressive stress, that is very prominent near the anvil end facing the hammer (red line in Fig. $7 \mathrm{~b}$ at about $\mathrm{t}=110 \mu \mathrm{s})$, corresponds to time of the maximum radial contraction (red line in Fig. $7 \mathrm{c}$ at about $\mathrm{t}=110 \mu \mathrm{s}$ ). Temporal double peak structure of the axial compressive wave (occurring at all locations, but becoming more prominent towards the steel-liquid $\mathrm{Pb}$ interface) develops as a combined result of: (i) anvil expansion, (ii) dilatation and shear waves originating from the surface and (iii) reflections from the liquid $\mathrm{Pb}$ back into the anvil. For the current geometry and materials effects (i) and (ii) are dominant, whereas reflections from the liquid $\mathrm{Pb}$ only slightly change pulse behavior (at least at the middle $\mathrm{r} / \mathrm{R}=0$ of the anvil). We will elaborate on this later in this section.

As stated earlier, one of the main objectives of the structural "Y"-code simulations is to obtain a detailed structure of the pressure pulse exerted upon liquid $\mathrm{Pb}$ by the current hammer-anvil system. To achieve that we extract the pressure history (given by Eq. 10) at the elements in the $\mathrm{Pb}$ right next to the solid-liquid interface at various radial positions across the piston face. This data, which is later used as boundary condition in acoustic/fluid dynamics simulations, is shown in Fig. 8 for the $50 \mathrm{~m} / \mathrm{s}$ impact. It can be seen that pressure pulse transmitted through the central part of the anvil is characterized by a distinctive double peak structure and has high degree of spatial non-uniformity. This double peak structure at the central part of the anvil face at the steel-liquid interface is an intrinsic feature of the nearfield stress state developing upon the longitudinal impact of the cylindrical bars and its shape is only slightly modified by the reflections across the interface, for the geometry and materials used in this study.

The above statement can be supported by examining the results obtained in simulation of a cylindrical projectile impacting a long cylindrical bar (no reflections from the end of the bar during times of interest) with $L_{\text {projectile }} / \mathrm{R}=1$ and stresses recorded at the axial position of $\mathrm{x} / \mathrm{R} \approx 1.2$ (see Fig. 9 ). It is worth noting that a specific structure of a stress wave propagating in a cylindrical bar upon impact of a cylindrical projectile made of the same material is solely predefined by the normalized length of a projectile $L_{\text {projectile }} / \mathrm{R}$ and normalized axial position along the bar $\mathrm{x} / \mathrm{R}$ at which pulse is recorded. For the current geometry, $L_{\text {anvil }} / \mathrm{R} \approx 1.2$ and the axial distance at which anvils contacts liquid $\mathrm{Pb}$ is $\mathrm{x} / \mathrm{R} \approx 1.2$. These values are very close to those used in a long bar impact simulation. In Fig. 9, time history of the axial $\sigma_{x x}$ and radial $\sigma_{r r}$ stress components obtained in the middle of the impacted bar $\mathrm{r}=0$ are plotted by blue and black lines, respectively. Also shown by 
March 7, $2017 \quad 16: 38$ WSPC/INSTRUCTION FILE

PressureWaves-ijam revised ${ }^{\circ} \mathrm{VS}{ }^{\circ}$ final

the green line is a time history of the radial displacement at the bar surface for the same axial position of $\mathrm{x} / \mathrm{R} \approx 1.2$. One can clearly see that the double peak structure of the axial stress component is very similar to that obtained for the current piston assembly at the solid-liquid interface (Fig. 8). It can be also observed that decrease in the amplitude of the compressive axial stress (first peak) correlates with the decrease in amplitude of the compressive radial stress. Once radial stress changes from compressive to tensile, amplitude of the compressive axial stress increases again until it attains its highest value (second peak) at the time of maximum tensile radial stress that also correlates with the time of maximum expansion of the bar due to the radial inertia.

$$
P=-\frac{1}{3}\left(\sigma_{x x}+\sigma_{y y}+\sigma_{z z}\right)
$$

\subsection{Pressure wave propagation in liquid $\mathrm{Pb}$}

In this section structure of the pressure wave as it propagates inside the sphere filled with liquid $\mathrm{Pb}$ is studied. A mon-linear acoustics solver based on the Westervelt equation and three different solvers of OpenFOAM ( "sonicLiquidFoam", "compressibleInterFoam" and "cavitatingFoam") are used to simulate wave propagation. All simulations in this section have been performed on $\frac{1}{7}$ section of a sphere with pressure pulse obtained in the structural "Y"-code simulations (see Fig. 8 for the $50 \mathrm{~m} / \mathrm{s}$ impact) applied as a time-dependent pressure boundary condition. The sphere is treated as fully rigid, such that pressure wave is entirely reflected from the boundary and all energy remains inside the $\mathrm{Pb}$. Simulations have been run for the 10 and $50 \mathrm{~m} / \mathrm{s}$ impact velocities. In all plots time $\mathrm{t}=0$ corresponds to the beginning of the impact. We start with the structure of the wave and comparison between the results obtained with different code for the case of $10 \mathrm{~m} / \mathrm{s}$ impact.

Structure of the wave in the horizontal plane (red plane in Fig. 3b) obtained with "sonicLiquidFoam" solver is shown in Fig. 10. Left and right columns of the figure show, respectively, pressure and velocity magnitude contours at different times during the wave propagation. From the plots in Fig. 10 the following observations can be made: (i) presence of perturbations on the wave front associated with the individual pulses at earlier times (e.g. parts a and b of the figure), (ii) merging of individual waves into a single combined wave propagating towards the center, (iii) visible imprint of fourteen distinct pistons during the entire propagation, (iv) some amplification of the maximum pressure and velocity at the late stages of propagation, but this amplification is significantly lower than that expected from a spherical (or cylindrical) geometrical convergence, (v) pressure and velocity at later times are characterized by a distinct narrow peak near the front edge of the pulse; (vi) presence of regions of "negative" pressure (allowed in "sonicLiquidFoam" solver) in the liquid mathrmPb in a wake of the pulse (Fig. 10g) indicating possible development of cavitating $\mathrm{Pb}$ regions. 
March 7, $2017 \quad 16: 38$ WSPC/INSTRUCTION FILE PressureWaves-ijam revised ${ }^{\circ} \mathrm{VS}{ }^{\circ}$ final

Structure of the wave in a vertical plane (blue plane in Fig. 3b) is shown by the pressure contours in Fig. 11. Results obtained with "compressibleInterFoam" and "Westervelt" solvers are presented in the left and right columns of the figure, respectively. One can see that the combined pressure wave has a complex spatial structure and its amplitude varies along the front edge. For the current structure of the pulse and arrangement of the pistons, pressure wave first reaches vertical axis at a certain distance (above and below) from the center of the sphere (Fig. 11 for times $\mathrm{t}=200$ and $250 \mu \mathrm{s})$. Non-uniformity of amplitude along the front edge means that initial interface velocity of the liquid-gas interface is also going to be non-uniform, as it is related to the strength of the wave at the moment it hits the interface.

When comparing results obtained with "compressibleInterFoam" and "Westervelt" solvers, one can see that the evolution of the wave structure as it propagates through the $\mathrm{Pb}$ is very similar (compare left and right columns in Fig. 11). It is interesting to note that the results of both solvers show development of very low pressure regions behind the pulse. The exact shape of those regions is slightly different, but the main features are the same. Pressure in those regions attempts to attain negative values during the computation, i.e. to put liquid into tension. Development of the negative pressure regions inside the liquid indicates possible development of cavitated $\mathrm{Pb}$ areas as a result of pulse propagation.

To get a detailed structure of the pressure wave and quantitatively compare results obtained with the different solvers, the instantaneous pressure profiles along several path lines have been extracted. Those path lines are marked by the green lines in Fig. 3b and denoted as "Profile Line 1", "Profile Line 2" and "Profile Line 3". "Profile Line 1" and "Profile Line 2" are inclined correspondingly by the angles $\pm 22.5^{\circ}$ relative to the horizontal plane and extend from the center of the piston patch and from in between two neighboring pistons toward the center of the sphere. "Profile Line 3" lays in a horizontal plane and spans from the wall of the sphere (in between three neighboring pistons) and the center.

Instantaneous pressure profiles along paths "Profile Line 1", "Profile Line 2" and "Profile Line 3" are shown correspondingly in Figs. 12, 13 and 14. At all plots horizontal axis corresponds to the radial position inside the sphere, where "0" corresponds to the center and " $0.5 \mathrm{~m}$ " to the wall of the sphere. Results are shown for the five different simulations: "Westervelt" acoustic solver with non-linear equation of state (red solid line), "sonicLiquidFoam" solver (black solid line), "cavitatingFoam" solver (cyan solid line), "compressibleInterFoam" (blue solid line) and simulation with extruded piston patch geometry run with "compressibleInterFoam" (blue broken line). Times in the simulation of the extruded piston patch geometry have been adjusted by the $25 \mu \mathrm{s}$ to account for how long it takes for the pulse to propagate through the extruded part. Starting with comparing the results obtained with different solvers one can clearly see that the pressure profiles along all path lines and at all times agree very well with each other. Minor differences observed near the rear edge of the pulse and behind the pulse can be attributed to the different numerical 
March 7, $2017 \quad 16: 38$ WSPC/INSTRUCTION FILE PressureWaves-ijam revised ${ }^{\circ} \mathrm{VS}{ }^{\circ}$ final

treatment of the areas where pressure attempts to attain negative values.

A good agreement between the results obtained with non-linear (Westervelt) and linear (OpenFOAM solvers) equation of state also means that at impact velocities around $10 \mathrm{~m} / \mathrm{s}$ non-linear effects associated with equation of state are negligible, and the linear barotropic equation of state performs well at this range. This also agrees with our preliminary estimations based on the amplitude of the density fluctuations. When comparing the results obtained for the extruded flat piston patch geometry (blue broken line) with those obtained for a curved piston patch aligned with a sphere (all solid lines) the effect of such geometry modification appears to be very small. It is also worth mentioning that using a hybrid (structured/unstructured) mesh in simulations with OpenFOAM solvers seems to work well and could be a good strategy to incorporate more complex geometry into simulations.

Following evolution of the pressure wave along different paths, a significant variation of temporal and spatial structure can be observed. One of the important things to note is that at later times (when wave approaches the free-surface if evacuated cavity is present inside the sphere), the pressure pulse is characterized by the narrow peak near the front edge of the pulse. Occurrence of this narrow peak of significant amplitude may cause liquid to cavitate very close to the free-surface (as a result of interaction between wave and the interface) and in the case of the initially imperfect interface may lead to the breakdown of the surface into a spray (see [Kedrinskii, 2005] for experiments and modeling of surface breakdown, phenomenon known as "sultans" in Russian literature).

Pressure profiles along paths "Profile Line 1", "Profile Line 2" and "Profile Line 3" for the high velocity $50 \mathrm{~m} / \mathrm{s}$ impact are shown in Figs. 15, 16 and 17, respectively. Results are presented for the four different simulations: "Westervelt" acoustic solver with non-linear equation of state (red solid line), "compressibleInterFoam" of OpenFOAM version $\mathrm{v}^{\mathrm{a}}$ with non-linear equation of state (Eq. 5) (black solid line), "Westervelt" acoustic solver with linear equation of state (red broken line) and "sonicLiquidFoam" of OpenFOAM version v4 with linear equation of state (Eq. 4) (black broken line). Results presented for the Westervelt solver and nonlinear equation of state have been obtained with parameter $\beta_{\mathrm{Pb}}$ (see section 2.2 for details) has been set to $\beta_{\mathrm{Pb}}=4$ (red solid lines in Figs. 12, 13, 14, 15, 16, 17). In simulations with linear equation of state $\beta_{\mathrm{Pb}}=1 . \beta_{\mathrm{Pb}}$ originates from Ballous statistical rule mentioned in Section 2.2. This value was confirmed using analysis based on Taits equation of state for liquid $\mathrm{Pb}$ [Salvatore, 2015].

One can see that also at velocity impact of $50 \mathrm{~m} / \mathrm{s}$ results obtained with inhouse "Westervelt" acoustic solver compare very well with those obtained with OpenFOAM solvers, along all paths and at all times for both non-linear and linear equation of state (compare solid red and black lines for non-linear equation of state and broken red and black lines for linear equation of state). It is also clear from

aDefault solver settings for "compressibleInterFoam" v4 have to be changed such that "transonic" option is turned on. 
March 7, $2017 \quad 16: 38$ WSPC/INSTRUCTION FILE PressureWaves-ijam revised ${ }^{\circ} \mathrm{VS}{ }^{\circ}$ final

the plots that non-linear wave propagation effects become significant at impact velocities of $50 \mathrm{~m} / \mathrm{s}$ and results obtained with non-linear and linear equation of state are rather different (compare solid and broken lines). Non-linearity manifests itself in a stronger steepening of the pulse front, increase in a wave propagation speed and redistribution of energy between the peaks. Considerable increase in the peak pressure at later times along paths "Profile Line 2" and "Profile Line 3" can be also observed in the simulations performed with non-linear equation of state. Stronger steepening of the pulse front followed by the sharp narrow peak obtained for $50 \mathrm{~m} / \mathrm{s}$ impact may further contribute to spallation of the free surface during the collapse of evacuated cavity.

\subsection{Dynamics of gas cavity collapse}

A collapse of a spherical gaseous target inside the prototype of the compression system is studied in this section with the main objective to test a numerical tool and its current limitations for simulating those kind of problems. As a start, evolution of initially smooth and perfectly symmetric cavity during the compression initiated by the simultaneous impact of the pistons is simulated. This is the first step toward exploring stability of the collapsing cavity under various scenario. A full 3D sphere has been simulated for the computational setup with extruded flat face piston patches (see Fig. 3b). Simulations have been carried out on a coarser mesh compared to those in section 3.2. Reduced mesh resolution had mild effect on the amplitude of the pressure pulse near the interface, but the overall structure of the wave for the low velocity impact of $10 \mathrm{~m} / \mathrm{s}$ remained the same. Results presented here have been obtained for the impact velocity of $10 \mathrm{~m} / \mathrm{s}$ and, for simplicity, a spherical gas cavity with radius $5 \mathrm{~cm}$ at the centre of the sphere.

When a combined converging pressure wave strikes the liquid-gas interface it is partially transmitted into the gas and partially reflected. The transmission ratio depends on the acoustic impedances of each fluid $z=\rho c$, where $\rho$ and $c$ are the density and sound speed of the fluid, respectively. In our case the pressure pulse is almost entirely reflected because of the severe mismatch between the acoustic impedances of liquid $\mathrm{Pb}$ and that of air. The reflected wave is a rarefaction wave that subjects the liquid to tension, which may cause development of cavitation regions. For a planar geometry and two fluids with a very large impedance ratio, the interface velocity can be approximated as:

$$
V_{\text {interface }} \approx \frac{2\left(P_{\max }-P_{0}\right)}{\rho_{\mathrm{Pb}} c_{\mathrm{Pb}}} \approx \frac{2 P_{\max }}{\rho_{\mathrm{Pb}} c_{\mathrm{Pb}}} .
$$

Initial interface velocity is then proportional to the maximum pressure (Eq. 11) and therefore, as spatially non-uniform wave arrives at the interface, different parts of the interface move at different velocities as a result of interaction with the pressure wave. This means that depending on the structure of the combined wave different shapes of the cavity can be achieved as it collapses. It should be noted that "compressibleInterFoam" solver used in those simulation is a two-phase solver only, so it 
March 7, $2017 \quad 16: 38$ WSPC/INSTRUCTION FILE

PressureWaves-ijam revised ${ }^{\circ} \mathrm{VS}{ }^{\circ}$ final

is not applicable if the details of cavitation regions formation and development are important. A three-phase solver (liquid $\mathrm{Pb}$, cavitating $\mathrm{Pb}$ and gas) with a cavitation model is to be used in this case. In the current simulations, when liquid goes under tension and pressure reaches predefined minimum, density starts to decrease and phase fraction $\alpha$ starts to decrease from " 1 " towards "0" indicating the phase change from liquid to gas. As such current numerical setup allows us to get some idea of where cavitation regions are likely to occur, but if cavitation process itself is of importance a more sophisticated cavitation model and three-phase solver are to be used. This is left for a future study.

Here the focus is on interface dynamics and surrounding liquid $\mathrm{Pb}$, whereas details of the gas behavior are omitted. Evolution of a spherical cavity during the compression for $10 \mathrm{~m} / \mathrm{s}$ impact is shown in Fig. 18 by the contours of velocity magnitude (left column) and density (right column) for the vertical cross-section plane. Also shown is a shape of the cavity (liquid-gas interface) that is plotted by the iso-surface of a phase fraction for $\alpha=0.5$. Parts (a) and (b) of the Fig. 18 correspond to the moment when pressure wave has just reached the free surface. At this moment no cavitation regions are observed in the $\mathrm{Pb}$ surrounding spherical target. As cavity getting compressed, formation of low density regions at some distance from the interface can be identified (Fig. 18d, f and h). Appearance of those regions indicates development of cavitation zones as the liquid is subjected to tension in those areas. As a volume of gas target continues to decrease and liquid $\mathrm{Pb}$ continues to expand into the space previously filled by the gas, low density regions become more obvious. In the current setup the amount of liquid is fixed, therefore the only way it can expand is by going into tension (reduce density) and eventually cavitate.

Following evolution of the velocity magnitude contours during the gas cavity collapse, one can see nearly spherical high velocity region surrounding the cavity. The size of this region seems to correlate with the radial distance from the interface at which low density regions start to appear. Similar results have been observed in our earlier work for a trapezoidal pulse compressing cylindrical gas cavity in the 2D cylindrical geometry [Suponitsky et al., 2014]. One can also see that initially spherical gas target changes its shape into oblate spheroid during the compression. This indicates non-uniform pressure distribution at the liquid-gas interface originating from the complex spatial structure of the converging wave. From the numerical point of view it is worth noting that the liquid-gas interface remains smooth and sharp when the radius of the cavity is bigger than unstructured mesh block in the center (up to $\mathrm{t}=575 \mu \mathrm{s}$ ). At later times surface ripples associated with transition from the structured orthogonal to unstructured tetrahedral mesh, can be observed. Some numerical artifacts at the transition region between structured and unstructured mesh can be also seen on the velocity magnitude contour plots at all times. 
March 7, $2017 \quad 16: 38$ WSPC/INSTRUCTION FILE PressureWaves-ijam revised ${ }^{\circ} \mathrm{VS}{ }^{\circ}$ final

\section{Conclusion}

Pressure wave propagation inside a pneumatic piston assembly and in liquid $\mathrm{Pb}$ have been studied numerically for the geometry and parameters of the experimental prototype of the plasma compression system constructed at General Fusion Inc.. Such set-up although in different geometries can be found in various engineering applications such as hydraulic bars, drilling hammers, water-hammer in pipelines etc. Thus the methodology and the underlying physics as described below is applicable beyond the particular application investigated in this study.

Structure of the pressure wave developing upon impact in the hammer-anvil assembly has been examined using structural mechanics research code "Y"-code. The obtained pressure pulse has been then propagated in the liquid $\mathrm{Pb}$ with several fluid solvers within OpenFOAM software and also with in-house non-linear acoustic solver based on the Westervelt equation. Some preliminary results for the compression of a spherical gas target have been also obtained. Main results and conclusions of this study are listed below:

- Pressure pulse generated by the current hammer-anvil assembly exhibits complex spatial and temporal structure, caused by the relative short aspect ratios of both the hammer and anvil pistons. Thus, besides the longitudinal waves predicted by the 1D impact bar analysis, transverse waves also appeared. This resulted in the distinctive double peak temporal structure of the pulse transmitted into the liquid and also in strong amplitude variation of the pressure across anvil face.

- Results of the wave propagation in the liquid $\mathrm{Pb}$ obtained with different solvers within OpenFOAM ( "sonicLiquidFoam", "cavitatingFoam" and "compressibleInterFoam") agree very well between each other and with inhouse non-linear acoustic solver based on the Westervelt equation. At impact velocity of $10 \mathrm{~m} / \mathrm{s}$ non-linear effects are insignificant and using linear equation of state is appropriate. At impact velocity of $50 \mathrm{~m} / \mathrm{s}$ non-linear effects can not be neglected and using a non-linear equation of state becomes essential. Due to the overall good agreement between the Westervelt equation and the CFD analysis, it can be concluded that accumulative non-linear propagation effects dominate over local non-linear effects, thus justifying simplification in modelling of such wave propagation.

- Development of cavitation regions around the gas cavities during compression has been observed at all impact velocities. Some cavitation spots have been also noted near the piston patches and in the wake of the pressure pulse. Such cavitation spots can cause structural damage on the anvil face.

- Interface dynamics during the collapse of a spherical gas cavity is captured exceptionally well by the compressible multiphase solver "compressibleInterFoam". As the liquid mass was fixed, zones of liquid going into tension (reduced density) were identified as it expanded into the gas cavity. This 
$\begin{array}{lllll}\text { March } & 7, & 2017 & 16: 38 & \text { WSPC/INSTRUCTION }\end{array}$

PressureWaves-ijam revised ${ }^{\circ} \mathrm{VS}{ }^{\circ}$ final

will eventually lead to cavitation as was also experimentally found. Using a hybrid mesh in OpenFOAM simulations produces good results, but for fine details a structured orthogonal mesh aligned with the interface is more suitable.

\section{References}

Avital, E. J., Musafir, R. E. and Korakianitis, T. [2013] "Nonlinear Propagation of Sound Emitted by High Speed Wave Packets," Journal of Computational Acoustics 21(3), 1-21.

Charriere, B., Decaix, J. and Goncalves, E. [2015] "A comparative study of cavitation models in a Venturi flow," European Journal of Mechanics - B/Fluids, Part A 49, 287-297.

Deshpande, S. S., Anumolu, L. and Trujillo, M. F. [2012] "Evaluating the performance of the two-phase flow solver interFoam," Comput. Sci. Disc. 5, 014016.

Don, V., Avital, E. J. and Motallebi, F. [2013] "Computational and Experimental Investigation of Supersonic Flow and their Controls," Proc World Academy Sci, Eng Tech 73, 1181-1187.

Ferziger, J. H., Peric, M. [1997] Computational Methods for Fluid Dynamics (Springer-Verlag, Berlin Heidelberg, Germany).

Hamilton, M. F. and Blackstock, D. T. [1998] Non-linear acoustics: Theory and Applications (Academic Press).

Huneault, J. and Higgins, A. [2015] "Imploding Shock-Driven Cavitation in Cylindrical Cavities," In Proceedings of the 30th International Symposium on Shock Waves, Tel-Aviv, Israel, July 19-24.

Ichihara, M., Ripepe, M., Goto, A., Oshima, H., Aoyama, H., Iguchi, M., Tanaka, K. and Taniguchi, H. [2009] "Airwaves generated by an underwater explosion: Implications for volcanic infrasound," J. Geophys. Res. 114, B03210, doi:10.1029/2008JB005792.

Johnsen, E. and Colonius, T. [2008] "Shock-induced collapse of a gas bubble in shockwave lithotripsy," J. Acoust. Soc. Am. 124(4), 2011-2020.

Kedrinskii, V.K. [2005] "Hydrodynamics of Explosion," Springer, Berlin.

Laberge, M. [2009] "Experimental results for an acoustic driver for MTF," J. Fusion Energy 28(2), 179-182.

Latham, J.P., Munjiza, A., Mindel, J., Xiang, J., Guises, R., Garcia, X., Pain, C., Gorman, G. and Piggott, M. [2008] "Modeling of massive particulates for breakwater engineering using coupled FEMDEM and CFD," Particuology 6, $572-583$.

Meyers, M. A. [1994] Dynamic Behavior of Materials (John Wiley \& Sons, Inc., New York).

Munjiza, A. and Andrews, K. R. F. [2000] "Penalty function method for combined finite-discrete element systems comprising large number of separate bodies," Int. J. Numer. Meth. Engng 49, 1377-1396. 
$\begin{array}{lllll}\text { March } & 7, & 2017 & 16: 38 & \text { WSPC/INSTRUCTION }\end{array}$

PressureWaves-ijam revised ${ }^{\circ}{ }^{2}{ }^{\circ}$ final

Munjiza, A., and Latham, J.P. [2004] "Some computational and algorithmic developments in computational mechanics of discontinua," Phil. Trans. R. Soc. London 8, 1817-1833.

Munjiza, A. [2005] The Combined finite-discrete method (John Wiley \& Sons, Ltd., England, UK).

Nemat-Nasser, S., Isaacs, J.B. and Starrett, J.E. [1991] "Hopkinson Techniques for Dynamic Recovery Experiments," Proceedings: Mathematical and Physical Sciences 435(1894), 371-391.

Punekar, J., Avital, E. J. and Musafir, R. E.[2010] "Computations of Nonlinear Propagation of Sound Emitted from High Speed Mixing Layers," Open Acoustics J. 3, 11-20.

Richardson, D., Froese, A., Suponitsky, V., Reynolds, M. and Plant, D. [2013] "Status of Progress Towards Acoustic Magnetized Target Fusion at General Fusion," In Proceedings of the 34th Annual Conference of the Canadian Nuclear Society, Toronto, Canada, June 9-12.

Rougier, E., Knight, E. E., Broome, S. T., Sussman, A. J. and Munjiza, A. [2014] "Validation of a three-dimensional Finite-Discrete Element Method using experimental results of the Split Hopkinson Pressure Bar test," International Journal of Rock Mechanics \& Mining Sciences 70, 101-108.

Rusche, H. [2002] "Computational fluid dynamics of dispersed two-phase flows at high phase fractions," Imperial college of science. London. UK: Technology $\&$ Medicine.

Salvatore, E. [2015] "Sound propagation in liquid metal for a nuclear fusion applications," M.Sc. Thesis, School of Engineering and Material Science, Queen Mary University of London, London, UK.

Suponitsky, V., Froese, A. and Barsky, S. [2014] "Richtmyer-Meshkov Instability of a Liquid-Gas Interface Driven by a Cylindrical Imploding Pressure Wave," Computers \& Fluids 89,1-19.

Suponitsky, V., Plant, D., Avital, E. J. and Munjiza, A. [2015] "Propagation of Pressure Waves in Compression System Prototype for Magnetized Target Fusion Reactor in General Fusion Inc.," In Proceedings of the 30th International Symposium on Shock Waves, Tel-Aviv, Israel, July 19-24.

Takayama, K. [1993] "Application of Underwater Shock Wave Focusing to the Development of Extracorporeal Shock Wave Lithotripsy," Japanese Journal of Applied Physics 32(5B).

Tsouvalas, A. and Metrikine, A.V. [2016] "Noise reduction by the application of an air-bubble curtain in offshore pile driving," Journal of Sound and Vibration 9, 150-170.

Vales, F., Moravka, S., Brepta, R. and Cerv, J. [1996] "Wave Propagation in a Thick Cylindrical Bar Due to Longitudinal Impact," JSME International Journal, Series $A$ 39(1), 60-70. 
March

7,

$2017 \quad 16: 38$

WSPC/INSTRUCTION

FILE

PressureWaves-ijam revised VS'final

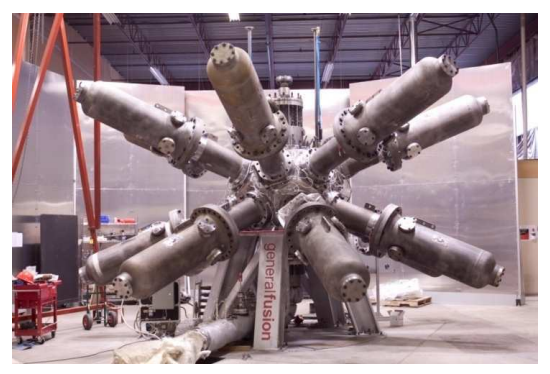

Fig. 1: Fourteen piston prototype of the compression system constructed in General Fusion Inc.

(a)

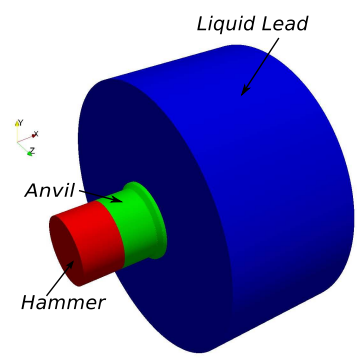

(b)

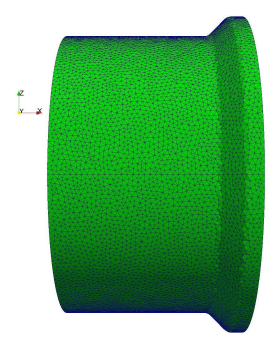

Fig. 2: (a) Computational model used to study impact of an isolated piston in "Y"- code simulations; (b) anvil piston (with flat face) along with tetrahedron computational mesh used in majority of simulations.
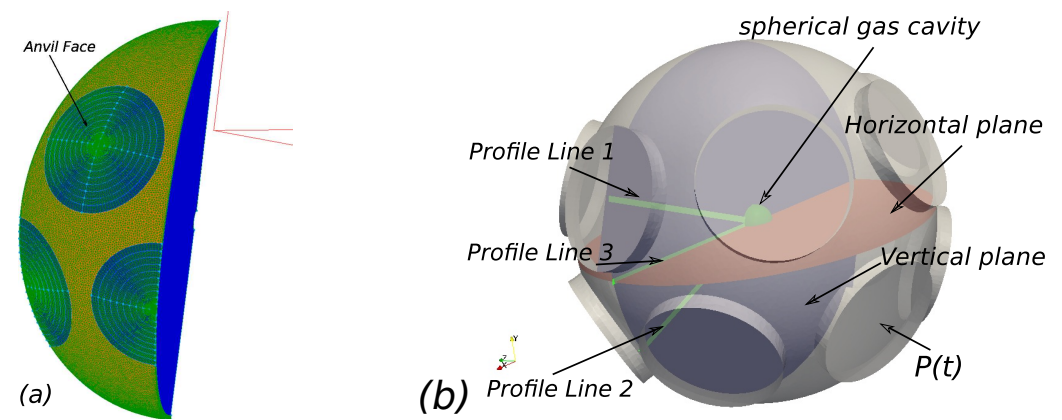

Fig. 3: (a) Computational model used in OpenFOAM simulations to study pressure wave propagation; (b) A schematic of a computational model with extruded piston patches to study cavity collapse dynamics. 
March

7

$2017 \quad 16: 38$

PressureWaves-ijam revised ${ }^{\circ}{ }^{2}{ }^{\circ}$ final

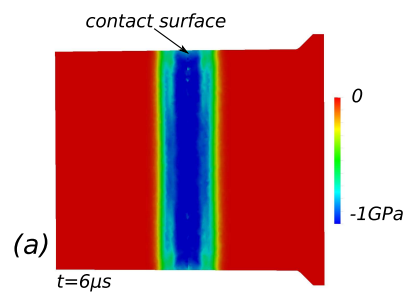

(b)

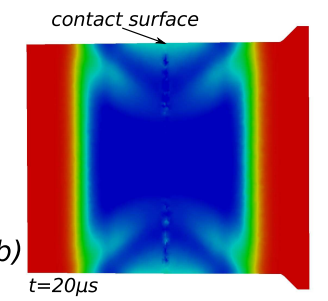

FILE
WSPC/INSTRUCTION

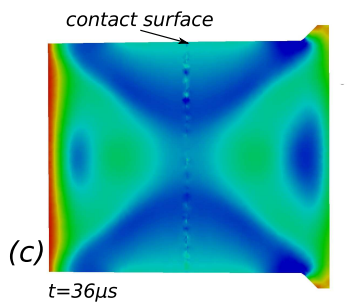

Fig. 4: Compressive wave shown by the contours of stress component $\sigma_{x x}$ (direction of wave propagation) in hammer-anvil system generated by $50 \mathrm{~m} / \mathrm{s}$ impact. At $\mathrm{t}=0$ hammer comes in contact with the anvil piston. (a) $C_{1} \mathrm{t} / \mathrm{R}=0.2$, (b) $C_{1} \mathrm{t} / \mathrm{R}=0.72$, (c) $C_{1} \mathrm{t} / \mathrm{R}=1.3$.
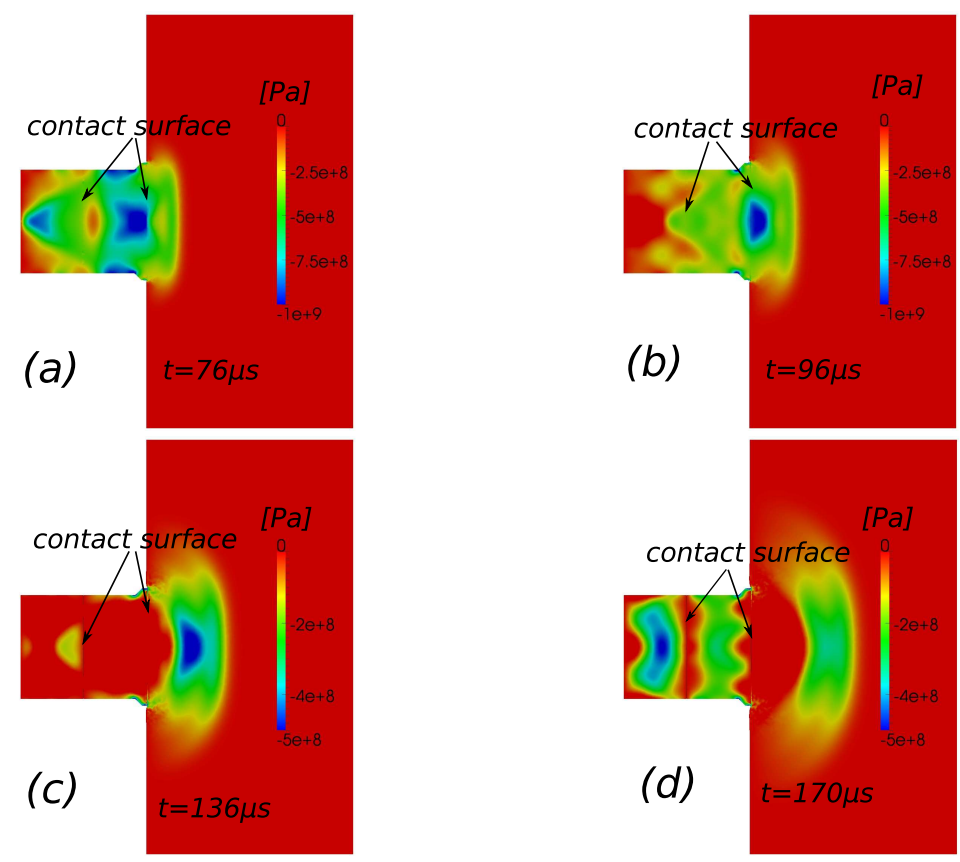

Fig. 5: Pressure wave transmission into the liquid lead tank shown by the contours of axial stress component $\sigma_{x x}$. Negative stress values correspond to material under compression. 
March $\quad 7, \quad 2017 \quad 16: 38 \quad$ WSPC/INSTRUCTION

PressureWaves-ijam revised ${ }^{\circ}$ VS'final

FILE

Pressure Wave in Liquid Generated by Pneumatic Pistons and its Interaction with a Free Surface
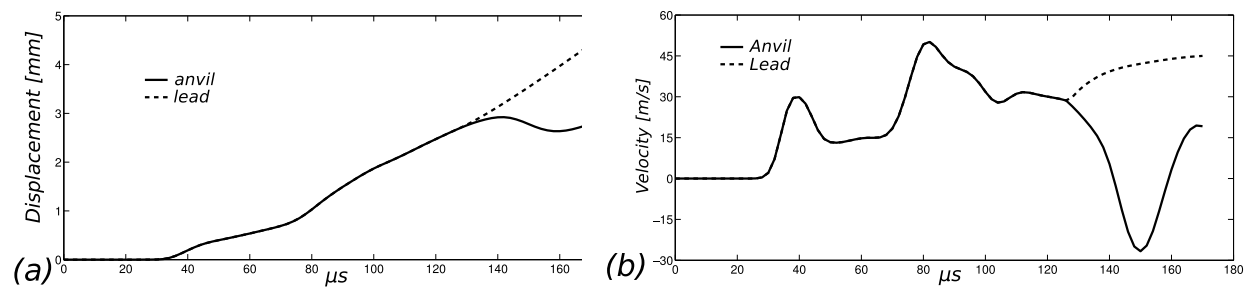

Fig. 6: Axial displacement (a) and axial velocity (b) at the steel-liquid $\mathrm{Pb}$ interface shown at $\mathrm{r} / \mathrm{R}=0$ (center of the anvil face). Solid and broken lines correspond, respectively, to the nodes of steel anvil and liquid lead across the interface. 
March

7
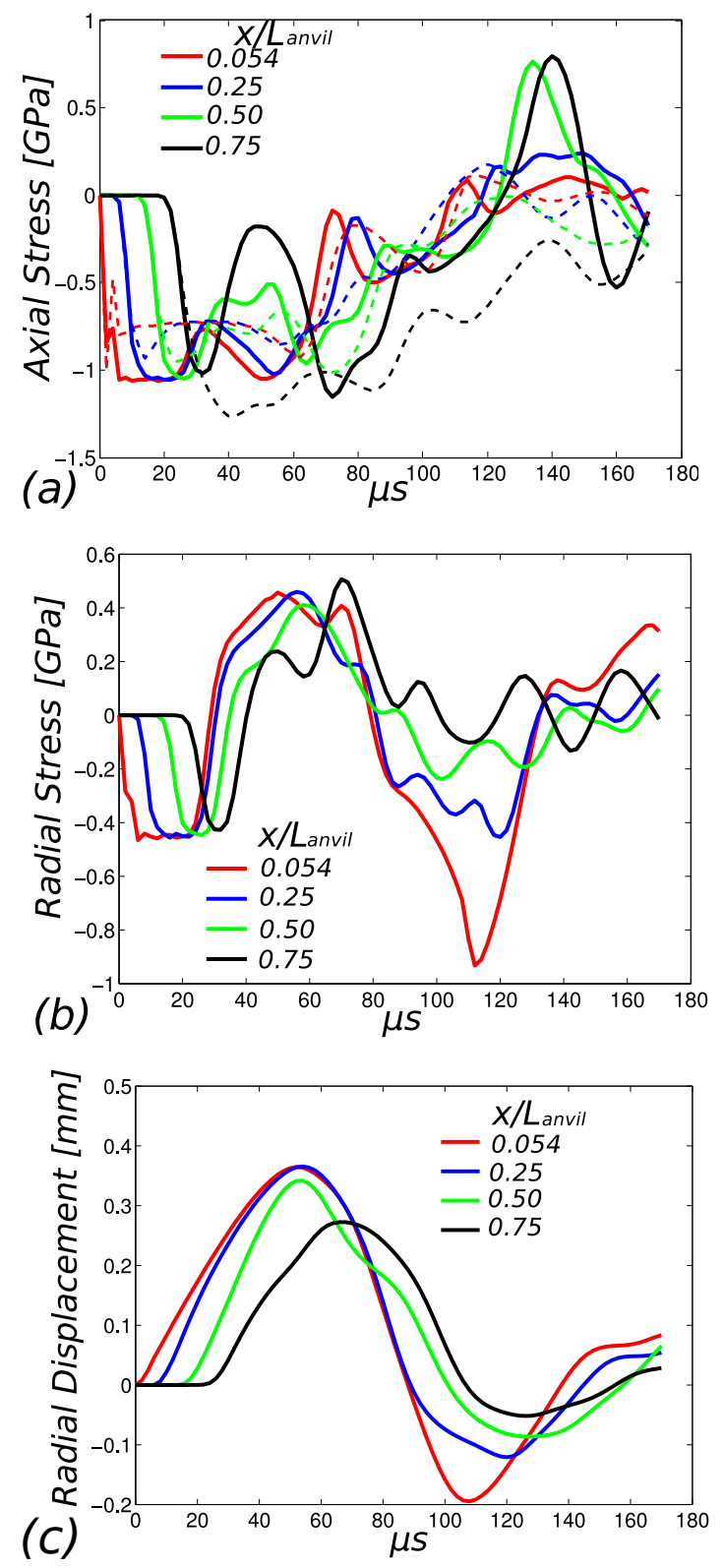

Fig. 7: Stress history (a) axial $\sigma_{x x}$ and (b) radial $\sigma_{r r}$ at several positions inside the anvil for $50 \mathrm{~m} / \mathrm{s}$ impact. Positions are normalized by the length of the anvil and $\mathrm{x}=0$ corresponds to the end facing the hammer. Solid lines show results at the center $\mathrm{r} / \mathrm{R}=0$. Broken lines added at part (a) show results at the edge $\mathrm{r} / \mathrm{R}=1$. 
$\begin{array}{lllll}\text { March } & 7, & 2017 & 16: 38 & \text { WSPC/INSTRUCTION }\end{array}$

PressureWaves-ijam revised ${ }^{\circ} \mathrm{VS}^{\prime}$ final

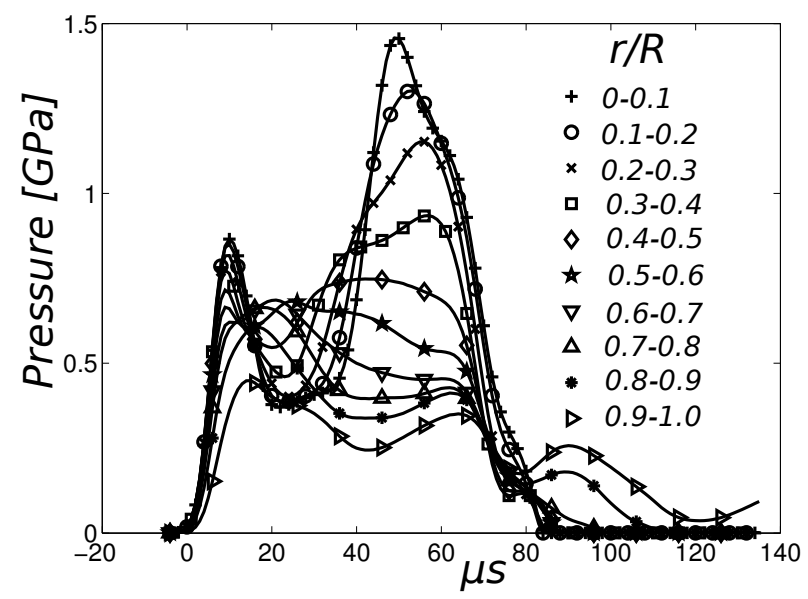

Fig. 8: Pressure pulse transmitted into liquid $\mathrm{Pb}\left(P=-\frac{1}{3}\left(\sigma_{x x}+\sigma_{y y}+\sigma_{z z}\right)\right)$ at different radial positions across the anvil face obtained in "Y"- code simulation and then applied as pressure boundary condition in fluid simulations. Impact velocity is $50 \mathrm{~m} / \mathrm{s}$.

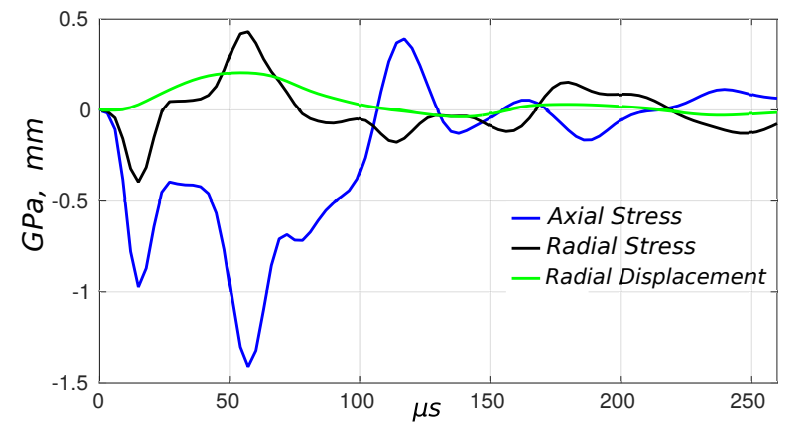

Fig. 9: Pressure pulse obtained from the simulation where steel projectile with $L_{\text {projectile }} / \mathrm{R}=1$ impacts a long steel bar of the same diameter when measured at the axial position of $\mathrm{x} / \mathrm{R}=1.2$ and $\mathrm{r}=0$. Impact velocity is $50 \mathrm{~m} / \mathrm{s}$. Blue and black lines correspond to the axial and radial stress components. Radial displacement at the anvil surface $r / R=1$ is plotted by the green line. 
March

7,

$2017 \quad 16: 38$

WSPC/INSTRUCTION

FILE

PressureWaves-ijam 'revised ${ }^{\circ} \mathrm{VS}^{\circ}$ final
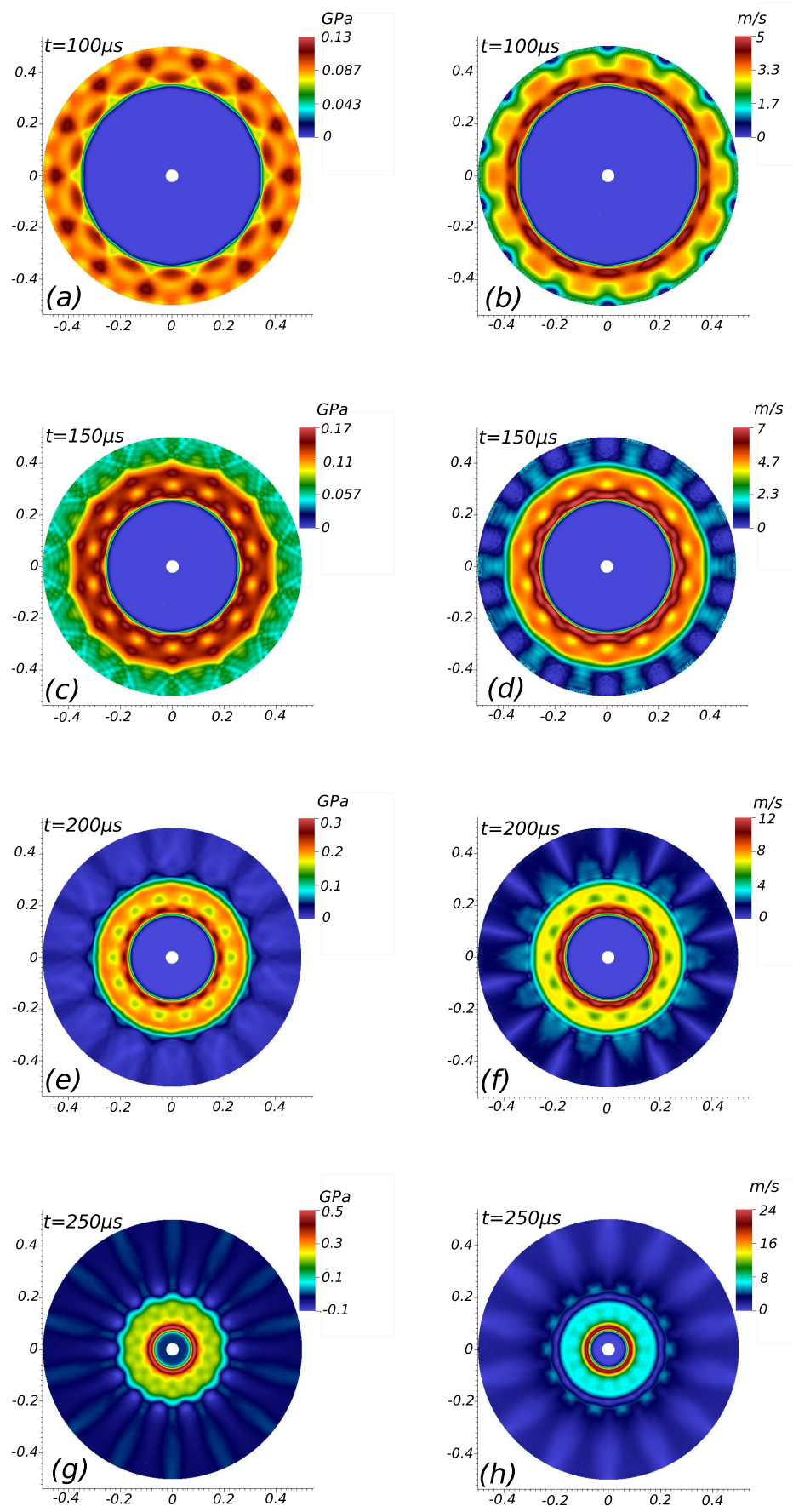

Fig. 10: Pressure (left column) and velocity magnitude (right column) contours of the converging pressure wave shown for the horizontal cross-section (see Fig. 3b) obtained with "sonicLiquidFoam". Impact velocity $10 \mathrm{~m} / \mathrm{s}$. 
March

7,

$2017 \quad 16: 38$

WSPC/INSTRUCTION

FILE

PressureWaves-ijam revised 'VS' final
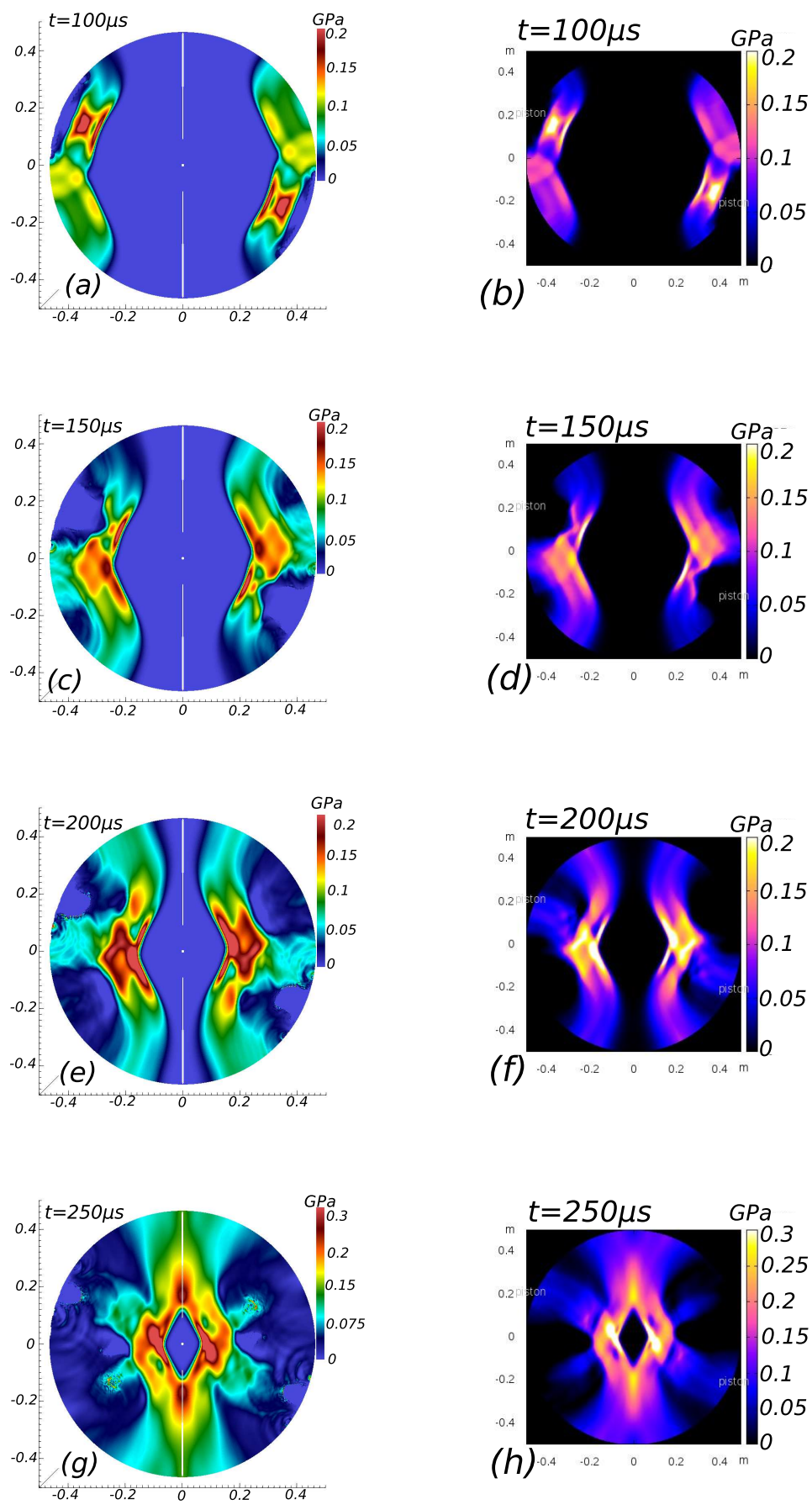

Fig. 11: Pressure contours of the converging wave shown for the vertical crosssection (see Fig. 3b). Left column - "compressibleInterFoam" solver, right column "Westervelt" solver. Impact velocity $10 \mathrm{~m} / \mathrm{s}$. 
$\begin{array}{lllll}\text { March } & 7, & 2017 & 16: 38 & \text { WSPC/INSTRUCTION }\end{array}$

PressureWaves-ijam revised ${ }^{\circ} \mathrm{VS}^{\prime}$ final

Suponitsky, Plant, Avital, Munjiza

(a)

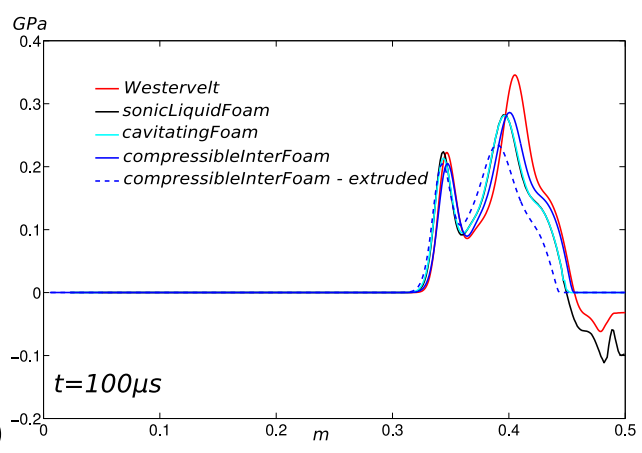

(b)
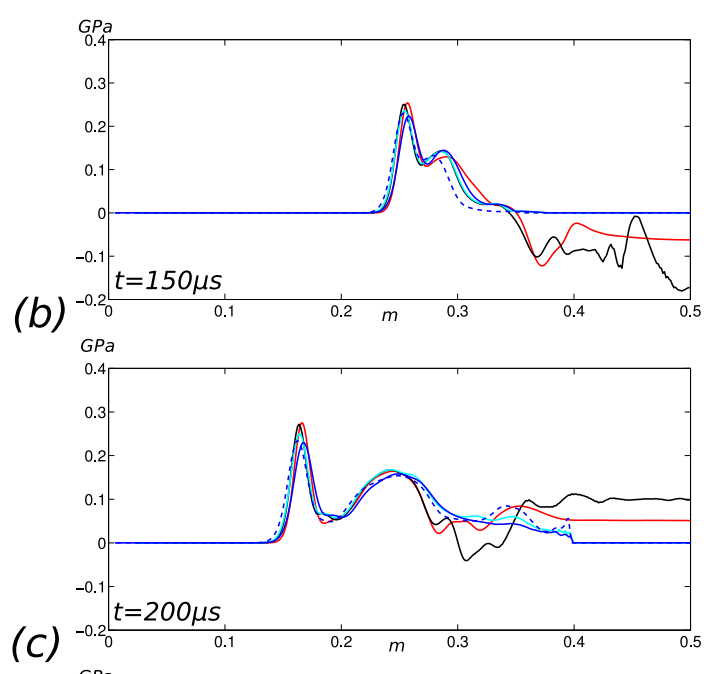

(C)

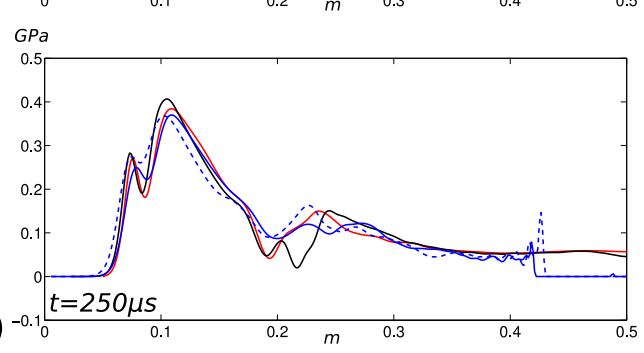

Fig. 12: Pressure profiles along path "Line 1" (see Fig. 3b). Path goes from center of the piston face towards the center of the sphere. Impact velocity $10 \mathrm{~m} / \mathrm{s}$. 
March $\quad 7, \quad 2017 \quad 16: 38$ WSPC/INSTRUCTION $\quad$ FILE

PressureWaves-ijam 'revised 'VS'final

Pressure Wave in Liquid Generated by Pneumatic Pistons and its Interaction with a Free Surface
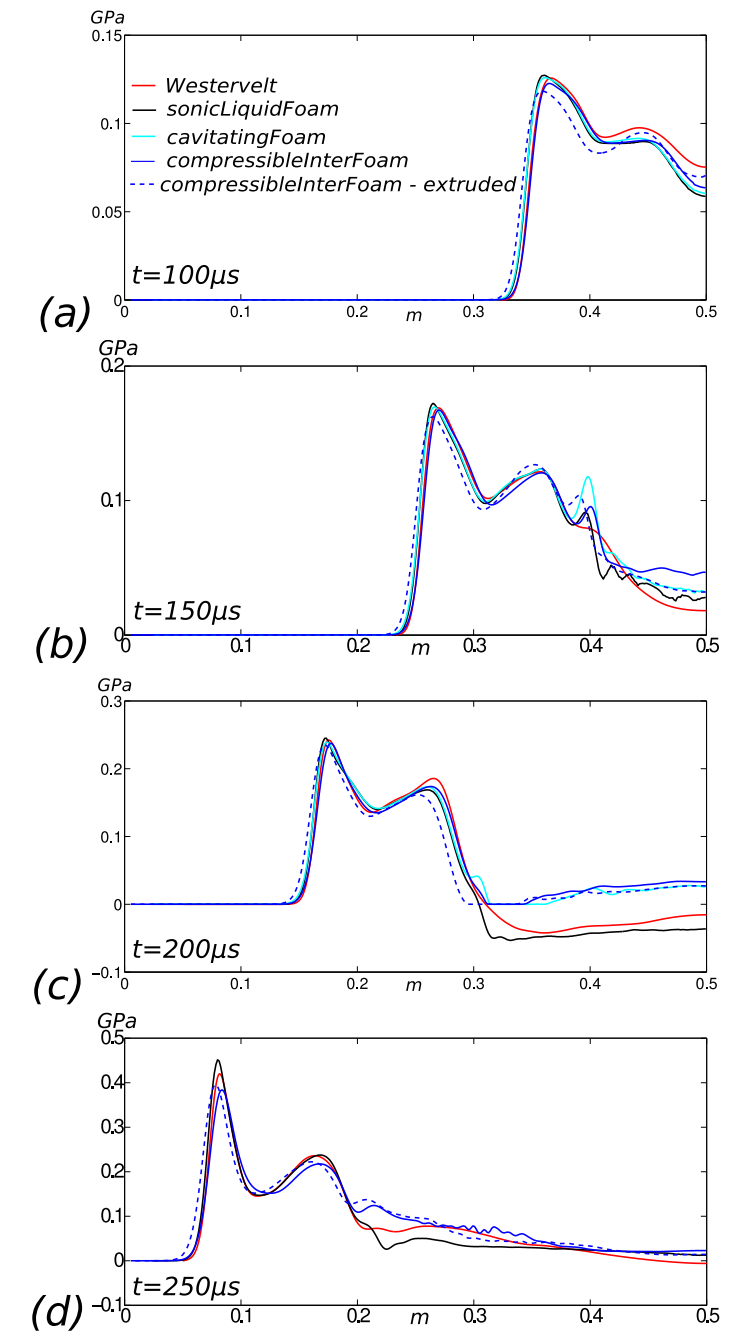

Fig. 13: Pressure profiles along the path "Line 2" (see Fig. 3b). (angle of $-22.5^{\circ}$ between the path and horizontal plane.) Path goes from in-between piston faces towards the center of the sphere. Impact velocity $10 \mathrm{~m} / \mathrm{s}$. 
$\begin{array}{llllll}\text { March } & 7, & 2017 & 16: 38 & \text { WSPC/INSTRUCTION } & \text { FILE }\end{array}$

PressureWaves-ijam revised ${ }^{\circ} \mathrm{VS}^{\prime}$ final

Suponitsky, Plant, Avital, Munjiza
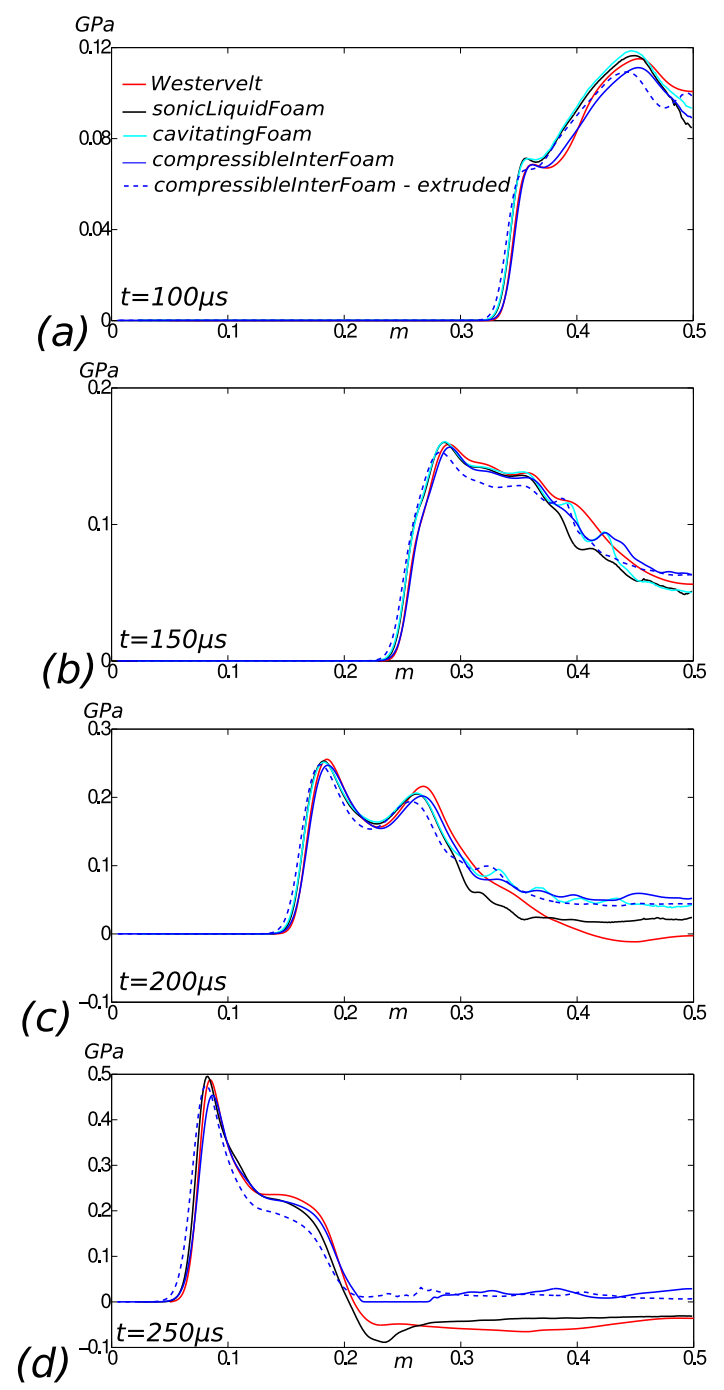

Fig. 14: Pressure profiles along the path "Line 3" (see Fig. 3b). Path follows horizontal plane from in-between piston faces towards the center. Impact velocity $10 \mathrm{~m} / \mathrm{s}$. 


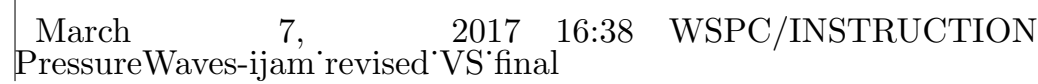

FILE

Pressure Wave in Liquid Generated by Pneumatic Pistons and its Interaction with a Free Surface
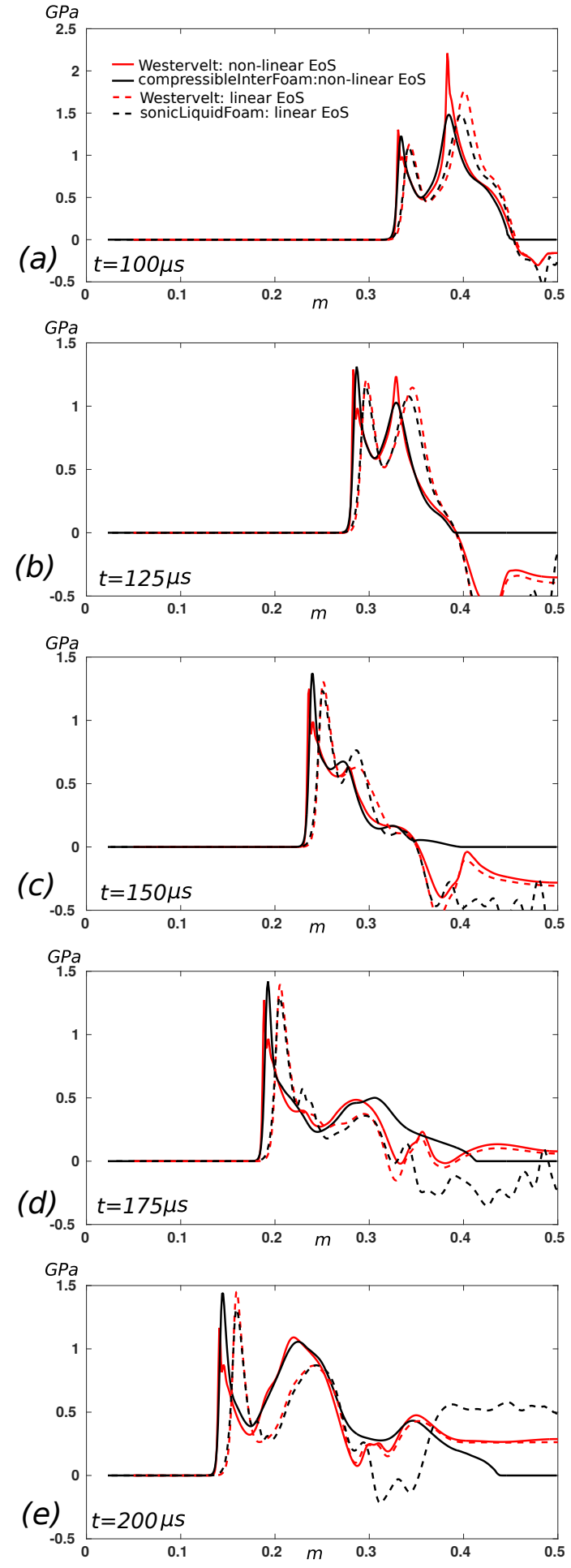

Fig. 15: Pressure profiles along the path "Line 1" (see Fig. 3b). Path goes from center of the piston face towards the center of the sphere. Impact velocity $50 \mathrm{~m} / \mathrm{s}$. 

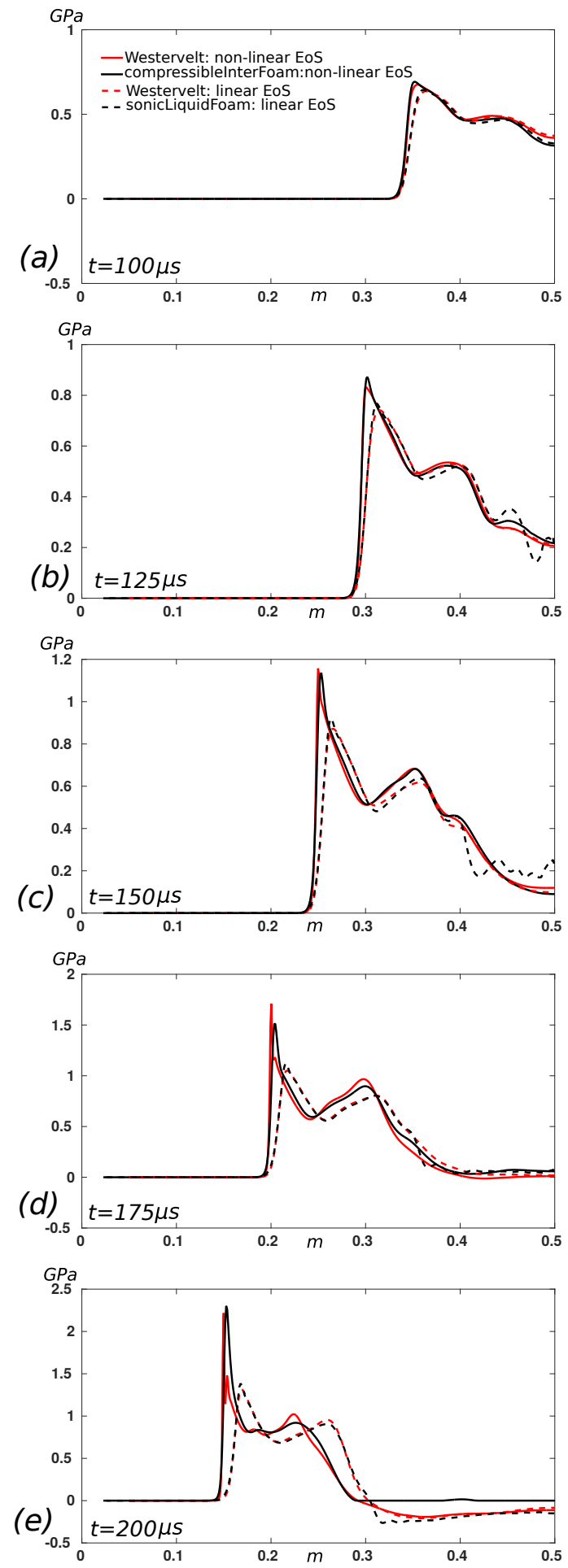

Fig. 16: Pressure profiles along the path "Line 2" (see Fig. 3b). (angle of $-22.5^{\circ}$ between the path and horizontal plane.) Path goes from in-between piston faces towards the center of the sphere. Impact velocity $50 \mathrm{~m} / \mathrm{s}$. 


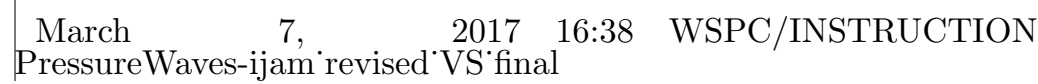

FILE
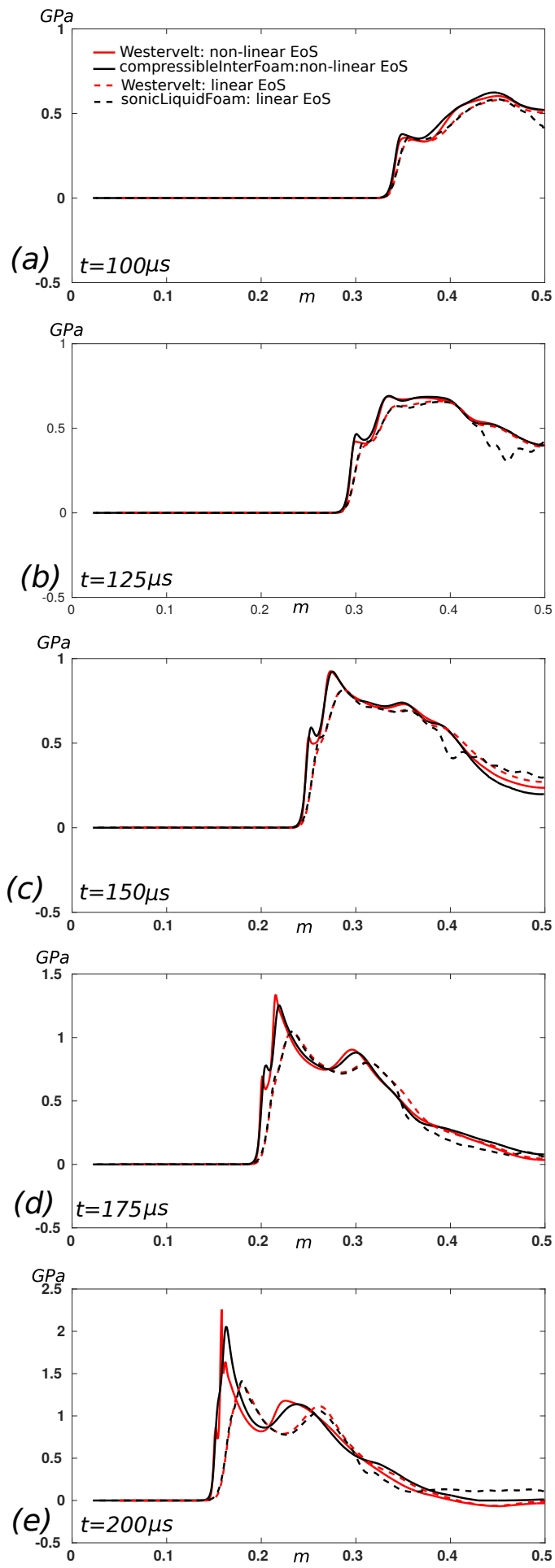

Fig. 17: Pressure profiles along the path "Line 3" (see Fig. 3b). Path follows horizontal plane from in-between piston faces towards the center. Impact velocity $50 \mathrm{~m} / \mathrm{s}$. 
March

7,

$2017 \quad 16: 38$

WSPC/INSTRUCTION

FILE

PressureWaves-ijam 'revised 'VS'final

34 Suponitsky, Plant, Avital, Munjiza
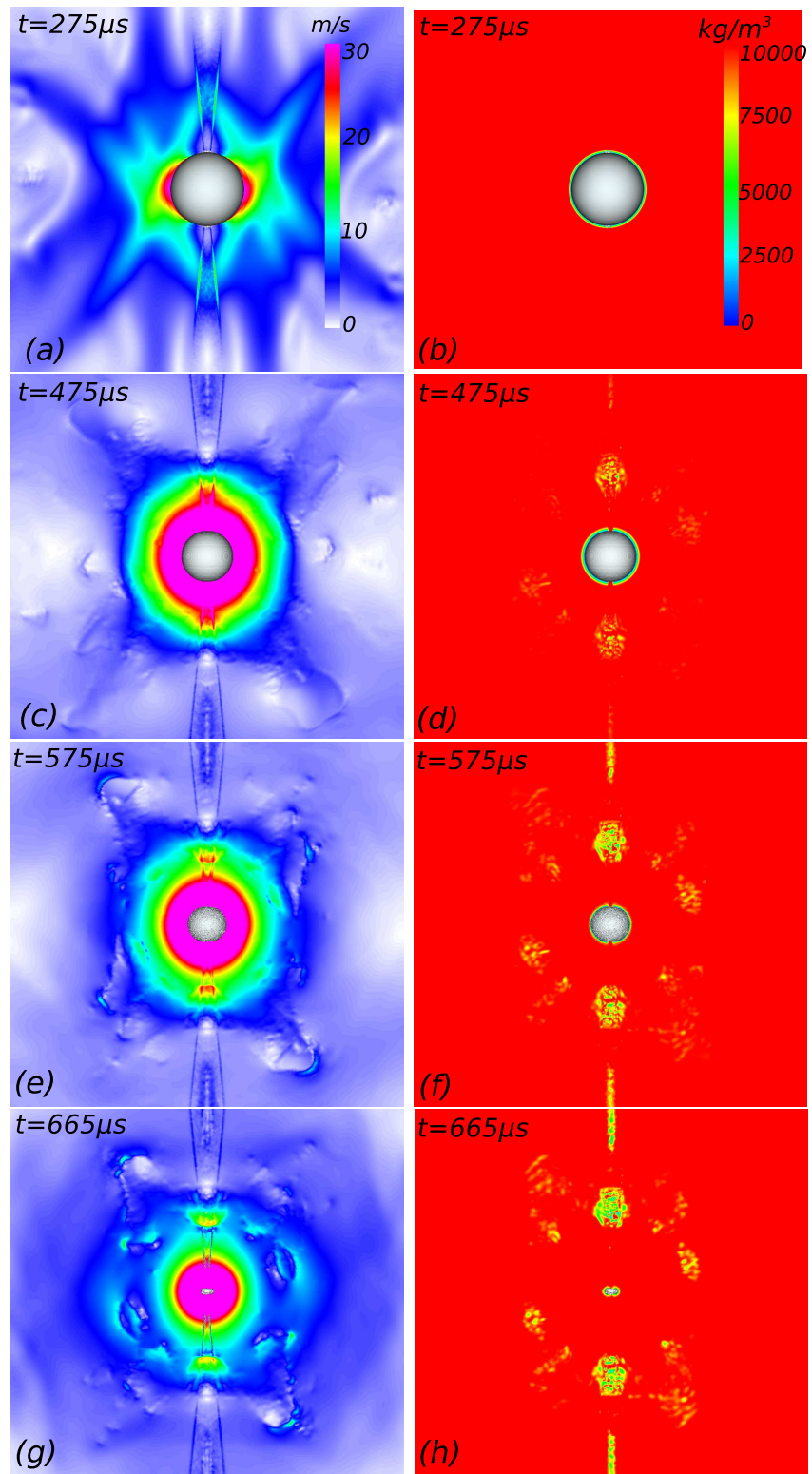

Fig. 18: Collapse of a spherical cavity obtained with "compressibleInterFoam" solver. Contours of the velocity magnitude and density are shown in left and right columns, respectively. Impact velocity $10 \mathrm{~m} / \mathrm{s}$. 